\title{
Turbulent viscosity optimized by data assimilation
}

\author{
Y. Leredde ${ }^{1}$, J.-L. Devenon ${ }^{2}$, I. Dekeyser ${ }^{1}$ \\ ${ }^{1}$ Centre d'Océanologie de Marseille, Université de la Méditerranée, Campus de Luminy-Case 901-F 13288 Marseille Cedex 9, France \\ e-mail: leredde@com.univ-mrs.fr \\ ${ }^{2}$ LSEET, Université de Toulon et du Var, BP 132-F 83957 La Garde Cedex, France
}

Received: 21 October 1998 / Revised: 5 July 1999 / Accepted: 20 July 1999

\begin{abstract}
As an alternative approach to classical turbulence modelling using a first or second order closure, the data assimilation method of optimal control is applied to estimate a time and space-dependent turbulent viscosity in a three-dimensional oceanic circulation model. The optimal control method, described for a 3-D primitive equation model, involves the minimization of a cost function that quantifies the discrepancies between the simulations and the observations. An iterative algorithm is obtained via the adjoint model resolution. In a first experiment, a $k+L$ model is used to simulate the one-dimensional development of inertial oscillations resulting from a wind stress at the sea surface and with the presence of a halocline. These results are used as synthetic observations to be assimilated. The turbulent viscosity is then recovered without the $k+L$ closure, even with sparse and noisy observations. The problems of controllability and of the dimensions of the control are then discussed. A second experiment consists of a two-dimensional schematic simulation. A 2-D turbulent viscosity field is estimated from data on the initial and final states of a coastal upwelling event.
\end{abstract}

Key words. Oceanography: general (numerical modelling) - Oceanography: physical (turbulence, diffusion, and mixing processes)

\section{Introduction}

In ocean models, the system of mean equations for momentum, temperature and salinity contain unknown second order correlations between fluctuations components of momentum and the buoyancy. Closure assumptions need to be introduced to relate frictional effects to the calculated large-scale velocity field, and the diffusive effects to the gradients of the temperature and salinity fields. These effects are produced in turbulence- like fashion by motions on scales too small to be resolved by the grid. These are called sub-grid scale phenomena. To close the system, the effects of these scales are usually parametrized in a simple way through the use of the eddy viscosity and diffusivity concept. Typically in the past, constant values of eddy viscosity (with different values in the horizontal and vertical directions) or empirical variable eddy viscosity, allowing non-linear effects to be more realistic, were used. More recently, with the increasing resolution capacities of present-day computers, the diffusion coefficients have often been determined from local values of scalar properties of the turbulence. Approaches of this type make use of one or more additional transport equations (Rodi, 1980). Today, while models have become quite sophisticated in computational and turbulence modelling aspects, they depend on numerous calibration parameters that must be extrapolated from limited field measurements. Furthermore, many uncertainties that cannot be accounted for by these turbulence models remain.

As an alternative approach, an inverse strategy is here proposed. In applied sciences, inverse methods are commonly used to extract useful inferences about the world from physical measurements (Menke, 1984). Here, the turbulent viscosity and diffusivity are fitted from data issuing from oceanographic observations. The method is a specific case of the optimal control method often called the adjoint method (Seiler, 1993). The ideas and mathematical concepts of optimal control theory were formalized about thirty years ago (Lions, 1971) and have since received much attention for applications in oceanography (Begis and Crepon, 1975; Devenon, 1990). The aim of the optimal control method is to find the best parameters of a model to simulate the computed values closest to those observed. The adjoint method has been most often used to fit initial conditions (Moore, 1991), but also boundary conditions (Lellouche et al., 1998), or specific parameters like the ocean surface heat fluxes (Roquet et al., 1993)). This variational method involves the minimization of a cost function which is the 
norm of the difference between the computed and observed values. An algorithm is obtained via the adjoint equations for the construction of the gradient of the cost function with respect to the parameters. Once the gradient has been determined, the minimization can be performed using any gradient descent algorithm.

The efficiency of the optimal control method to optimize time-constant viscosity distributions in 1-D vertical models has already been underlined ( $\mathrm{Yu}$ and O'Brien, 1991; Panchang and Richardson, 1993). In these studies, initial and boundary conditions are assumed to be well known, the variational formulation of the dynamic model acting as a strong constraint. In the case of 1-D advection-diffusion equation (Leredde et al., 1998) it has been shown that, with such a strong constraint formulation, the simultaneous optimization of the initial conditions, the boundary conditions and the space and time distributed viscosity coefficient becomes rather difficult. This is due to the fact that a single solution for all the controls is not ensured if no penalization of the first guesses of the controls is included in the cost function. Instead of using this type of strong constraint formulation, Eknes and Evensen (1997) have developed a weak constraint formulation which allows the observational data, the turbulent vertical viscosity, the initial and boundary conditions and also the model equations to be affected by errors. This approach involves a sophisticated iterative representer method (Bennett, 1992) and generates a highly non-linear problem whose solution depends on the required first guesses of the control and the error weights. Eknes and Evensen (1997) have used a 1-D Ekman model which is only an approximation of the primitive equations. Their results indicate that model deficiencies, such as neglected physics, are accounted for through the weak constraint formulation to ensure an inverse solution in agreement with the measurements. In this respect and in the framework of this non-exact model, their fit is closer to the data than those obtained by the strong constraint formulation (Yu and O'Brien, 1991).

In the present study, a 3-D primitive equation model has been adopted. Since the model is expected to be more exact and the representer method is not yet implemented in this three-dimensional case, the strong constraint variational formulation is used. Furthermore, in previous studies (Yu and O'Brien, 1991; Panchang and Richardson, 1993; Eknes and Evensen, 1997), the turbulent viscosity was taken to be constant in time. In fact, as mentioned many times in the literature (e.g. Blumberg and Goodrich, 1990), turbulent mixing is time-dependent especially for transient phenomena such as wind-driven or tidal processes. Therefore, the optimal control method is formalized in the more general frame of the optimization of a turbulent viscosity which can vary in the four space and time dimensions.

This induces a large number of parameters to be optimized requiring at least the same number of data to be assimilated. We have thus chosen to create synthetic observations with a complete turbulence model. The chosen model is a $k+L$ model (Leendertse et al., 1973;
Leendertse and Liu, 1977) using a single transport equation for the turbulent kinetic energy $k$ and an algebraic formulation for a mixing length scale $L$. It allows the construction of a data set and of the associated turbulent viscosity set to be recovered by the inverse method.

The physical and mathematical background is given in Sect. 2, including the description of the 3-D multilayer dynamic model and the adjoint model resulting from the variational formalism. This section deals with the complete and continuous optimization problem and adopts a more general framework than the applications which are made in Sects. 3 and 4 . In addition to the numerical computational cost, it becomes rather difficult to solve optimization problems which have a size equal to the number of grid points and time steps in the discretized form. Therefore, the first applications of our data assimilation model concern fairly simple oceanographic processes allowing the numerical treatment of the method with an acceptable space-time dimension.

In Sect. 3, a wind-driven mixing event of a stratified water column inducing inertial oscillations is simulated by the 3-D multilayer dynamical model with open lateral boundaries and consequently the process is 1-D. Section 4 deals with the optimization of a 2-D space-dependent turbulent viscosity in the case of a wind-driven mixing event of coastal upwelling. Concluding remarks are presented in Sect. 5.

\section{Tools}

\subsection{The circulation model}

The direct model (Leendertse et al., 1973), recently implemented (Lellouche, 1995) is a classical primitive equation model for coastal seas. Many others are based on a similar approach (e.g. Nihoul, 1977; Thouvenin and Salomon, 1984; Van Dam and Louwersheimer, 1990). The Boussinesq approximation (density is constant except in the buoyancy term, (Pedlosky, 1982)), and those of hydrostasy and $f$-plane are made. The physical state of the fluid is given by the seven equations for the average variables:

$$
\begin{aligned}
& \frac{\partial u}{\partial t}+\frac{\partial(u u)}{\partial x}+\frac{\partial(u v)}{\partial y}+\frac{\partial(u w)}{\partial z}-f v+\frac{1}{\rho_{0}} \frac{\partial p}{\partial x} \\
& -\frac{v_{h}}{\rho_{0}}\left(\frac{\partial^{2} u}{\partial x^{2}}+\frac{\partial^{2} u}{\partial y^{2}}\right)-\frac{1}{\rho_{0}}\left(\frac{\partial}{\partial z}\left(v_{t} \frac{\partial u}{\partial z}\right)\right)=0 \\
& \frac{\partial v}{\partial t}+\frac{\partial(v u)}{\partial x}+\frac{\partial(v v)}{\partial y}+\frac{\partial(v w)}{\partial z}+f u+\frac{1}{\rho_{0}} \frac{\partial p}{\partial y} \\
& \quad-\frac{v_{h}}{\rho_{0}}\left(\frac{\partial^{2} v}{\partial x^{2}}+\frac{\partial^{2} v}{\partial y^{2}}\right)-\frac{1}{\rho_{0}}\left(\frac{\partial}{\partial z}\left(v_{t} \frac{\partial v}{\partial z}\right)\right)=0 \\
& \frac{\partial p}{\partial z}=-\rho g \\
& \frac{\partial u}{\partial x}+\frac{\partial v}{\partial y}+\frac{\partial w}{\partial z}=0
\end{aligned}
$$




$$
\begin{aligned}
& \frac{\partial S}{\partial t}+\frac{\partial(u S)}{\partial x}+\frac{\partial(v S)}{\partial y}+\frac{\partial(w S)}{\partial z} \\
& -v_{h}^{S}\left(\frac{\partial^{2} S}{\partial x^{2}}+\frac{\partial^{2} S}{\partial y^{2}}\right)-\frac{\partial}{\partial z}\left(v_{t}^{S} \frac{\partial S}{\partial z}\right)=0 \\
& \frac{\partial T}{\partial t}+\frac{\partial(u T)}{\partial x}+\frac{\partial(v T)}{\partial y}+\frac{\partial(w T)}{\partial z} \\
& \quad-v_{h}^{T}\left(\frac{\partial^{2} T}{\partial x^{2}}+\frac{\partial^{2} T}{\partial y^{2}}\right)-\frac{\partial}{\partial z}\left(v_{t}^{T} \frac{\partial T}{\partial z}\right)=0 \\
& \rho=\rho_{0}\left(1+\alpha_{S}\left(S-S_{0}\right)+\alpha_{T}\left(T-T_{0}\right)\right)
\end{aligned}
$$

In this set of equations, $u, v, w$ are the mean velocity components in the three space dimensions $x, y, z ; t$ denotes the time, $p$ the pressure, $S$ the salinity, $T$ the temperature, $\rho$ the sea-water density. $\rho_{0}, T_{0}, S_{0}$ are constant reference values for density, temperature and salinity. $f$ is the Coriolis parameter, $g$ the gravitational acceleration, $\alpha_{S}$ the saline contraction coefficient, $\alpha_{T}$ the thermal expansion coefficient. $v_{h}, v_{t}$ are the horizontal and vertical turbulent viscosity coefficients. $v_{h}^{S}, v_{t}^{S}, v_{h}^{T}$ and $v_{t}^{T}$ are the turbulent diffusivity coefficients. A first approach, the zero order turbulence closure, consist of giving constant values to these viscosity and diffusivity coefficients without using additional transport equations.

When needed, heat fluxes at the surface and bottom and surface stresses can be introduced. In the following, only wind stress and bottom stress are considered and modelled.

At the upper boundary, the horizontal wind stress vector $\vec{\tau}$ can be estimated by the quadratic expression:

$\vec{\tau}=C_{d} \rho_{a}\left\|\vec{U}_{w}\right\| \vec{U}_{w}$

where $\rho_{a}$ is the air density, $\vec{U}_{w}$ the wind speed vector at $10 \mathrm{~m}$ above mean sea surface and $C_{d}$ a drag coefficient.

The bottom stress vector $\vec{\tau}_{b}$ is also expressed in the same way:

$\vec{\tau}_{b}=\rho_{b} g \frac{\left\|\vec{U}_{b}\right\| \vec{U}_{b}}{C^{2}}$

where $\rho_{b}$ is the water density at the bottom layer, $\vec{U}_{b}$ is the horizontal velocity vector at the bottom layer and $C$ the Chezy coefficient, homogeneous with $g^{\frac{1}{2}}$. For the numerical implementation (Lellouche, 1995), a finite difference approximation has been used both in time and space according to a vertical multilevel geometrical discretization.

In a first model version, used for open boundary condition optimizations (Lellouche et al., 1998), the turbulent viscosity and diffusivity coefficients were all set to constant values. A simple study of sensitivity of the model to the horizontal viscosity and diffusivity coefficients values shows that the horizontal diffusion terms are helpful to damp down the small-scale instabilities. Nevertheless a first approximation given by constant horizontal coefficients seems to be sufficient. The horizontal diffusion terms in Eqs. (1), (2), (5) and (6) were already written under this hypothesis.

In contrast, the vertical turbulent viscosity and diffusivities have a real influence on the numerical results, particularly for stratified seas, and must be specified with more accuracy.

\subsection{The $k+L$ turbulence model}

A classical approach consists in using a turbulence model where the turbulent viscosity and diffusivity coefficients are expressed as functions of the turbulent state of the sea water. This turbulent viscosity and diffusivity concept gives rise to various models. Among these models, the length scale formulation (Blackadar, 1962) can be chosen and might be corrected to take correctly into account the stratification effects. This dependence can be introduced via the Richardson number (Munk and Anderson, 1948). To go further, second order formulations (Rodi, 1980; Mellor and Yamada, 1982), known as $k-\varepsilon$ and $k-L$ models, have became rather popular, notably in the coastal and estuaries oceanographic community, and satisfy operational numerical needs. This operational goal restricts the implementation of higher order formulations with transport equations for Reynolds stresses (Mellor and Yamada, 1974; Andre et al., 1979). In coastal zones, recent works (Nihoul et al., 1989. Luyten et al., 1996) have shown that cruder models with only one transport equation for the turbulent kinetic energy ( $k$-models) could be sufficiently efficient. In the framework of this study and in order to progress towards our assimilation aims, a $k$-model, initially developed by Leendertse and Liu (1977), has been chosen. This model, called $k+L$ model, takes the following form.

The vertical eddy viscosity coefficient $v_{t}$ is evaluated by using a buoyancy extended Prandtl-Kolmogorov hypothesis:

$v_{t}=C_{v} L \sqrt{k} \exp (-m R i)$

where $L$ is a mixing length scale, $k$ the turbulent kinetic energy, and $m$ and $C_{v}$ positive constants.

$R i$ is the "turbulent" (not the "gradient") Richardson number.

$R_{i}=-\frac{g}{\rho_{0}} \frac{\partial \rho}{\partial z} \frac{L^{2}}{k}$

The mixing length scale is given by an algebraic function of the distance to the bottom $z^{*}$.

$L=\kappa z^{*} \sqrt{1-\frac{z^{*}}{d}}$

where $\kappa$ is the Von Kármán constant and $d$ the local water column height. The vertical eddy diffusivities for heat and salinity transport are assumed to be proportional to the turbulent vertical viscosity:

$v_{t}^{T}=\frac{v_{t}}{P r} \quad$ and $\quad v_{t}^{S}=\frac{v_{t}}{S c}$

where $P r, S c$ are respectively the turbulent Prandtl and Schmidt number. The turbulent kinetic energy is governed by the following transport equation: 


$$
\begin{gathered}
\frac{\partial k}{\partial t}+\frac{\partial(u k)}{\partial x}+\frac{\partial(v k)}{\partial y}+\frac{\partial(w k)}{\partial z}-v_{h}^{k}\left(\frac{\partial^{2} k}{\partial x^{2}}+\frac{\partial^{2} k}{\partial y^{2}}\right) \\
-\frac{\partial}{\partial z}\left(v_{t}^{k} \frac{\partial k}{\partial z}\right)-P-G+\varepsilon=0
\end{gathered}
$$

where $v_{h}^{k}$, the horizontal turbulent diffusivity for $k$, is considered as constant, and $v_{t}^{k}$, the vertical turbulent diffusivity for $k$, is assumed to be proportional to the turbulent viscosity:

$v_{t}^{k}=\frac{v_{t}}{\sigma_{k}}$

where $\sigma_{k}$ is also often called the turbulent Prandtl number for the turbulent kinetic energy.

Inside the fluid, the shear production of turbulent energy $P$ is expressed by:

$P=v_{t}\left(\left(\frac{\partial u}{\partial z}\right)^{2}+\left(\frac{\partial v}{\partial z}\right)^{2}\right)$

The bottom layer friction is taken into account by an additional production term:

$P=g\left(u_{b}^{2}+v_{b}^{2}\right)^{3 / 2} / C^{2}$

where $C$ is the Chezy coefficient previously introduced.

In the top layer, the wind and waves effects give also rise to an additional term:

$P=\alpha\left\|\vec{U}_{w}\right\|^{3}$

where $\alpha$ is a non dimensionless $\left(\mathrm{m}^{-1}\right)$ positive constant.

The buoyant production $G$ is modelled according to

$G=-\alpha_{S} v_{t}^{S} g \frac{\partial S}{\partial z}-\alpha_{T} v_{t}^{T} g \frac{\partial T}{\partial z}$

Instead of solving a transport equation which involves more model assumptions than the corresponding equation for $k, \varepsilon$, the turbulent kinetic energy dissipation is modelled according to

$\varepsilon=\varepsilon_{0} \frac{k^{3 / 2}}{L}$

where $\varepsilon_{0}$ is a positive constant.

\subsection{The optimal control formulation}

Even if the applications presented in Sects. 3 and 4 are two dimensional (1D space and 1D time or 2D space) and in order to adopt a more general framework, the complete $4 \mathrm{D}$ formalism is here described. The optimal control aims at finding the best parameters of the model to simulate computed values closest to those observed. It is exemplified here when the turbulent viscosity coefficient set is considered as the control to be optimized.

The physical state is described by the vector $Y=(u, v, w, p, S, T, \rho)$, solution of the set of differential Eqs (1)-(7) which can be re-written:

$\frac{\mathrm{d} Y}{\mathrm{~d} t}=F(Y, v) \quad$ in $\Omega \times(0, \tau)$ where $F$ is a non-linear operator, $\Omega$ is the space domain and $(0, \tau)$ is the time interval. $\Omega$ is considered in this general development as three dimensional. Here, $v=\left(v_{t}, v_{t}^{S}, v_{t}^{T}\right)$ is the only control of the system. In a more general case, the control could be the initial conditions (Le Dimet and Talagrand, 1986), the boundary conditions (Lellouche et al., 1994) or other tunable parameters such as friction coefficients (Begis and Crepon, 1975), optimized separately or together (Devenon, 1990; Leredde et al., 1998). In the following, in order to simplify somewhat the problem the turbulent Prandtl and Schmidt numbers, respectively $P r$ and $S c$, are set to constant values. Without restraining the scope of the present study, taking $P r=S c=1$, the turbulent diffusivities for heat and salt transport will be equal to the turbulent vertical viscosity. The control is then restricted to $v_{t}$ as a space and time dependent variable. In this general framework, $v_{t}$ is a function of the three space dimension $x, y, z$ and the time dimension $t$.

The observations of $Y$ being designated as $\hat{Y}$, a cost function which measures the mean squared discrepancy between the model solution and the observations can be defined as:

$J\left(Y\left(v_{t}\right), \hat{Y}\right)=\frac{1}{2}<Y-\hat{Y}, C_{\hat{Y}}^{-1} \cdot(Y-\hat{Y})>_{\Omega \times(0, \tau)}$

where $<,>_{\Omega \times(0, \tau)}$ is the inner product in $L^{2}(\Omega \times(0, \tau))$, this space being defined as the set of square integrable functions over $\Omega \times(0, \tau)$. $C_{\hat{Y}}$ is the covariance operator associated with the observations. Penalty terms could be added to this functional formulation. The convergence of the following method is then improved by the recall to a "first guess" of the control (Yu and O'Brien, 1991) or a smoothing term of the distributed control (Panchang and Richardson, 1993).

The optimal control $v_{t}$ opt minimizes the cost function $J$. The optimal control set cannot be obtained directly. It must be reached by successive minimization of $J$ through a descent algorithm. A classical way consists in computing the gradient of $J$ relative to the control. Introducing the adjoint state $Y^{*}$ (Lions, 1971), the expression of the gradient is:

$\nabla J\left(Y\left(v_{t}\right), \hat{Y}\right)=-\left(\frac{\partial F}{\partial v_{t}}\right)^{a d j} Y^{*}$

and the adjoint state verifies that:

$$
\frac{\mathrm{d} Y^{*}}{\mathrm{~d} t}+\left(\frac{\partial F}{\partial Y}\right)^{a d j} Y^{*}=C_{\hat{Y}}^{-1}\left(Y\left(v_{t}\right)-\hat{Y}\right) \text { in } \Omega \times(0, \tau)
$$

where adj denotes the adjoint operator. The details of the method for deducing the adjoint model can be found in Leredde et al. (1998) for the case of a one dimensional Burger's equation and in Leredde (1999) for these primitive equations and will not be presented further here. From Eq. (24), using the proper definition for $F$ (Eqs. 1-7), the adjoint state $Y^{*}=\left(u^{*}, v^{*}, w^{*}, p^{*}, S^{*}, T^{*}, \rho^{*}\right)$ verifies the adjoint equations: 


$$
\begin{aligned}
& \frac{\partial u^{*}}{\partial t}+2 u \frac{\partial u^{*}}{\partial x}+v\left(\frac{\partial u^{*}}{\partial y}+\frac{\partial v^{*}}{\partial x}\right)+w \frac{\partial u^{*}}{\partial z}-f v^{*} \\
& +S \frac{\partial S^{*}}{\partial x}+T \frac{\partial T^{*}}{\partial x}+\frac{v_{h}}{\rho_{0}}\left[\frac{\partial^{2} u^{*}}{\partial x^{2}}+\frac{\partial^{2} u^{*}}{\partial y^{2}}\right] \\
& +\frac{1}{\rho_{0}}\left[\frac{\partial}{\partial z}\left(v_{t} \frac{\partial u^{*}}{\partial z}\right)\right]=\frac{u-\hat{u}}{\sigma_{\hat{u}}^{2}} \\
& \frac{\partial v^{*}}{\partial t}+2 v \frac{\partial v^{*}}{\partial y}+u\left(\frac{\partial v^{*}}{\partial x}+\frac{\partial u^{*}}{\partial y}\right)+w \frac{\partial v^{*}}{\partial z}+f u^{*} \\
& +S \frac{\partial S^{*}}{\partial y}+T \frac{\partial T^{*}}{\partial y}+\frac{v_{h}}{\rho_{0}}\left[\frac{\partial^{2} v^{*}}{\partial x^{2}}+\frac{\partial^{2} v^{*}}{\partial y^{2}}\right] \\
& \quad+\frac{1}{\rho_{0}}\left[\frac{\partial}{\partial z}\left(v_{t} \frac{\partial v^{*}}{\partial z}\right)\right]=\frac{v-\hat{v}}{\sigma_{\hat{v}}^{2}} \\
& \frac{\partial p^{*}}{\partial z}-\frac{1}{\rho_{0}}\left(\frac{\partial u^{*}}{\partial x}+\frac{\partial v^{*}}{\partial y}\right)=\frac{p-\hat{p}}{\sigma_{\hat{p}}^{2}} \\
& \frac{\partial w^{*}}{\partial z}+u \frac{\partial u^{*}}{\partial z}+v \frac{\partial v^{*}}{\partial z}+S \frac{\partial S^{*}}{\partial z}+T \frac{\partial T^{*}}{\partial z}=\frac{w-\hat{w}}{\sigma_{\hat{w}}^{2}} \\
& \frac{\partial S^{*}}{\partial t}+u \frac{\partial S^{*}}{\partial x}+v \frac{\partial S^{*}}{\partial y}+w \frac{\partial S^{*}}{\partial z}+\rho_{0} \alpha_{S} \rho^{*} \\
& \quad+v_{h}^{S}\left[\frac{\partial^{2} S^{*}}{\partial x^{2}}+\frac{\partial^{2} S^{*}}{\partial y^{2}}\right]+\left[\frac{\partial}{\partial z}\left(v_{t}^{S} \frac{\partial S^{*}}{\partial z}\right)\right]=\frac{S-\hat{S}}{\sigma_{\hat{S}}^{2}} \\
& +u[P]^{*}=\frac{\partial T^{*}}{\partial x}+v \frac{\partial T^{*}}{\partial y}+w \frac{\partial T^{*}}{\partial z}+\rho_{0} \alpha_{T} \rho^{*} \\
& \frac{\sigma_{\hat{\rho}}^{2}}{\partial z}
\end{aligned}
$$

$\sigma_{\hat{y}_{i}}^{2}$, is the variance of the observation $\hat{y}_{i}$ corresponding to the variable $y_{i}$ in the case of statistical independence between data measurements. In fact, $\sigma_{\hat{y}_{i}}^{2}$, is the inverse of the weigh given to each observation. For example, if no temperature data is available, the weight is zero, $\sigma_{\hat{T}}^{2}$ is taken to be a large number and so there is no forcing term in Eq. (30).

Keeping this notation $y_{i}$ for any of the seven state variables, the dimensionless cost function can be written.

$J\left(Y\left(v_{t}(x, y, z, t)\right)\right)=\frac{1}{\tau \Omega} \int_{0}^{\tau} \int_{\Omega} \sum_{i=1}^{7} \frac{\left(y_{i}-\hat{y}_{i}\right)^{2}}{\sigma_{\hat{y}_{i}}^{2}} \mathrm{~d} \Omega \mathrm{d} t$

The expression of the gradient is then given by:

$$
\begin{aligned}
\nabla J\left(Y\left(v_{t}(x, y, z, t)\right), \hat{Y}\right)= & \frac{1}{\rho_{0}} \frac{\partial u}{\partial z} \frac{\partial u^{*}}{\partial z}+\frac{1}{\rho_{0}} \frac{\partial v}{\partial z} \frac{\partial v^{*}}{\partial z} \\
& +\frac{\partial S}{\partial z} \frac{\partial S^{*}}{\partial z}+\frac{\partial T}{\partial z} \frac{\partial T^{*}}{\partial z}
\end{aligned}
$$

If the turbulent viscosity is not fully time and space dependent, this general expression is integrated on the domain where the turbulent viscosity is constant. In Sect. 3, $v_{t}$ is considered as horizontally constant and the temperature is not taken into account. The expression of the gradient of the cost function relative to $v_{t}(z, t)$ becomes

$$
\begin{aligned}
\nabla J\left(Y\left(v_{t}(z, t)\right), \hat{Y}\right)= & \frac{1}{\Gamma} \int_{\Gamma}\left(\frac{1}{\rho_{0}} \frac{\partial u}{\partial z} \frac{\partial u^{*}}{\partial z}+\frac{1}{\rho_{0}} \frac{\partial v}{\partial z} \frac{\partial v^{*}}{\partial z} .\right. \\
& \left.+\frac{\partial S}{\partial z} \frac{\partial S^{*}}{\partial z}\right) d \Gamma
\end{aligned}
$$

where $\Gamma$ is the horizontal domain. In Sect. $4, v_{t}$ is a function of two space dimensions $x$ and $z$. The only assimilated data are temperature ones and the expression of the gradient of the cost function relative to $v_{t}(x, z)$ is

$\nabla J\left(Y\left(v_{t}(x, z)\right), \hat{Y}\right)=\frac{1}{\tau L} \iint \frac{\partial T}{\partial z} \frac{\partial T^{*}}{\partial z} \mathrm{~d} y \mathrm{~d} t$

where $L$ is the domain of variation of $y$. In fact, the general expression (33) can be adapted for each new configuration. For example, without the salinity term and with a time integration, the gradient expression of previous studies (Yu and O'Brien, 1991; Panchang and Richardson, 1993; Eknes and Evensen, 1997) could be found.

Once the gradient has been computed, the minimization of the cost function can be performed using any gradient descent algorithm. Here, a classical quasiNewton method (Gillbert and Lemarechal, 1989) is used.

For the numerical implementation, this continuous formulation must be converted to the discretized one. The way to proceed is not straightforward and involves theoretical and technical developments (Lellouche et al., 1998).

\section{Viscosity optimization}

\subsection{Description of the experiment}

Although the numerical tools described in the previous section should allow the treatment of a fully three dimensional case of a rotating fluid, a simple physical 1$\mathrm{D}$ case is investigated as a first attempt. The 3-D model is then used with open lateral boundaries at which Neumann conditions are imposed and all the variables are taken to be horizontally uniform. In this academic framework, the observation data set is created using the $k+L$ model described in Sect. 2.2. This numerical construction of error free data is usually done to check the results of new assimilation methods (Courtier and Talagrand, 1990). The aim is to recover these "simulated" data by means of the full identification of the turbulent viscosity coefficients. It means that a zero order turbulence closure version of the model is used instead of solving the $k+L$ closure.

In order to illustrate this 1-D optimization problem, the turbulent mixing of the water column resulting from a wind energy input at the sea surface is studied. The 
direct simulation consists in modelling the development of inertial oscillations in the presence of a halocline break. An 8-day simulation is performed with a time step of 20 min and a vertical grid size of $2 \mathrm{~m}$. The model is started from a state of rest considering a simplified two-layer water column with fresh water $(10 \mathrm{~m}$ thick, $0 \mathrm{psu})$ upon salty water $(20 \mathrm{~m}$ thick, $30 \mathrm{psu})$. The wind blows along the $x$ direction and its speed increases linearly from $5 \mathrm{~m} \mathrm{~s}^{-1}$ to $10 \mathrm{~m} \mathrm{~s}^{-1}$ during the simulation. The solution of the computation is given in Fig. 1 . Inertial oscillations remain trapped in the upper layer of the water column until the mechanical mixing energy, resulting from the downward diffusion of a fraction of the energy input by wind, overcomes the stabilizing effect of the stratification. Then, the halocline is suddenly broken and the motion can diffuse downward and the salinity upward. In the following, these simulated data are used as observations to be assimilated by the optimal control method. An initial model solution (Fig. 2) from which to start the minimizing procedure is computed with a constant value of $10 \mathrm{~cm}^{2} \mathrm{~s}^{-1}$ arbitrarily chosen for the turbulent viscosity. One can remark that this value is not accounted for as a first guess in the cost function as it is needed for a weak constraint formulation (Eknes and Evensen, 1997) to ensure a single solution (Bennett and Miller, 1991). In a strong constraint formulation, even though it can be used to accelerate the convergence of the method $(\mathrm{Yu}$ and O'Brien, 1991), it is not required if the problem remains over-determined (Menke, 1984). In any case, such a first guess choice could be difficult in this case of timedependent viscosity.

A comparison between the observation simulation (Fig. 1) and the initial simulation (Fig. 2) shows that the solution of the model is highly controlled by the turbulent viscosity. This latter is about zero at the halocline that acts as a physical wall for the diffusion. As the wind speed increases, the eddy viscosity increases to a threshold value inducing the sudden halocline break. The oscillation amplitude and damping rate follow this time-dependence of the viscosity. As this viscosity varies in the whole space and time domain $\Omega \times(0, \tau)$, the dimension of the control vector is equal to $M \times N$ where $M$ is the number of grid points and $N$ is the number of time steps. In order to keep the problem over-determined, the number of data to be assimilated must overtake the control dimension. All the model solution variables, here $u, v, S$ can be taken as observations. Different numerical experiments using different data sets are performed. The variances of the selected data are then set to a finite value corresponding to the inverse weights given to each observation. The other data variances are set to infinity expressing the complete lack of any other available information. The forcing (a)

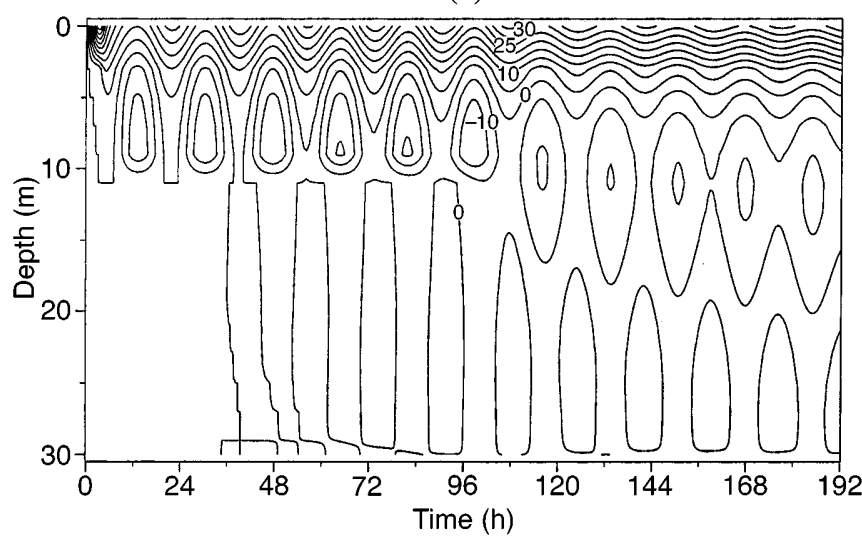

(c)

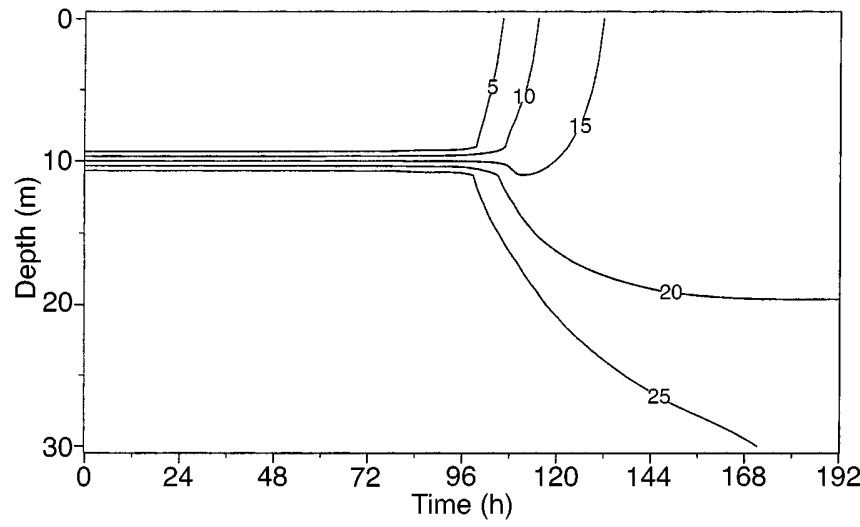

(b)

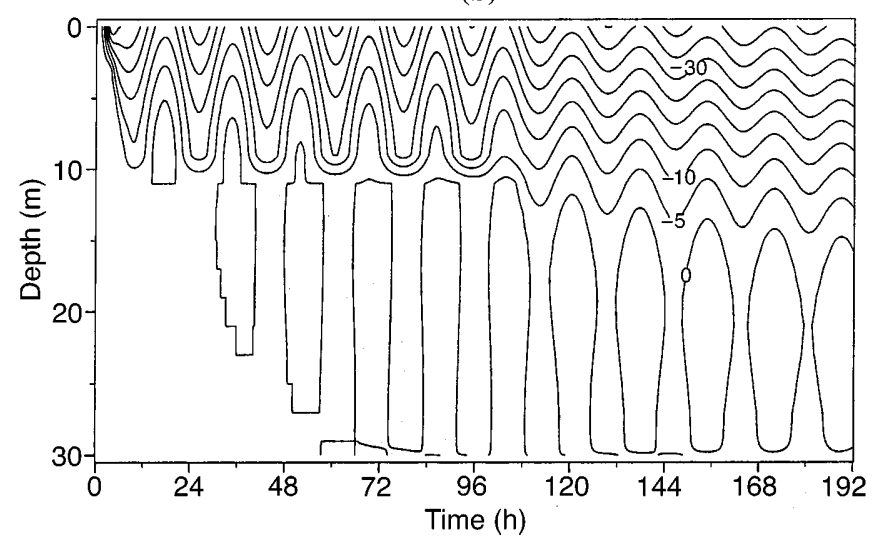

(d)

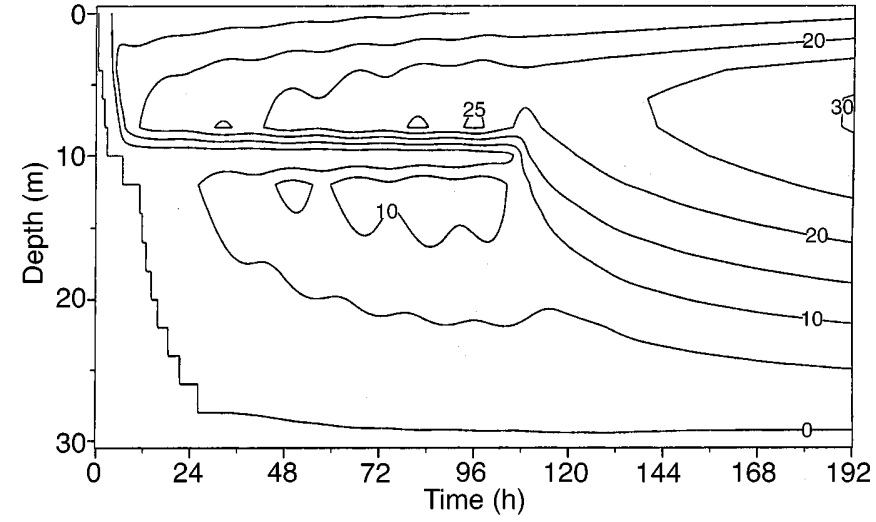

Fig. 1a-d. Solution of the direct simulation used to construct the observations. a Velocity $u\left(\mathrm{~cm} \cdot \mathrm{s}^{-1}\right), \mathbf{b}$ velocity $v\left(\mathrm{~cm} \cdot \mathrm{s}^{-1}\right), \mathbf{c}$ salinity $(\mathrm{psu}), \mathbf{d}$ turbulent viscosity $\left(\mathrm{cm}^{2} \cdot \mathrm{s}^{-1}\right)$. The contour intervals are 5 units for each variable plotted 
(a)

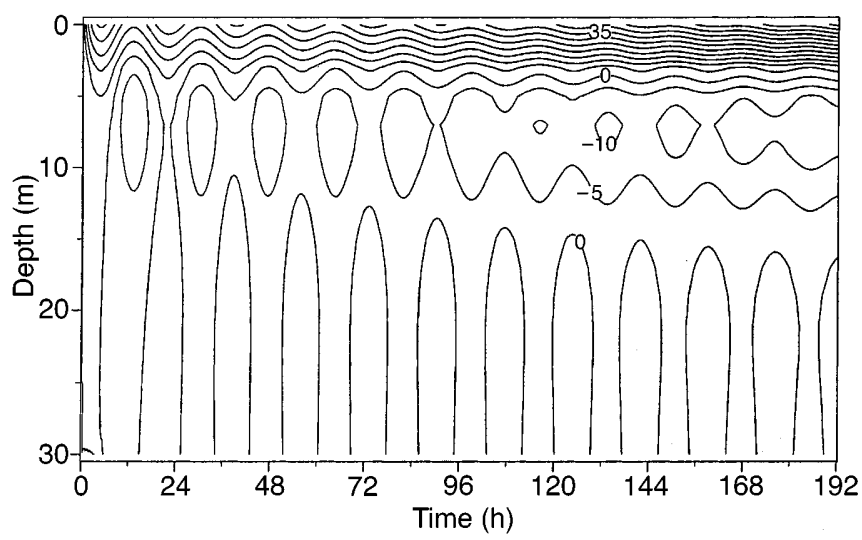

(b)

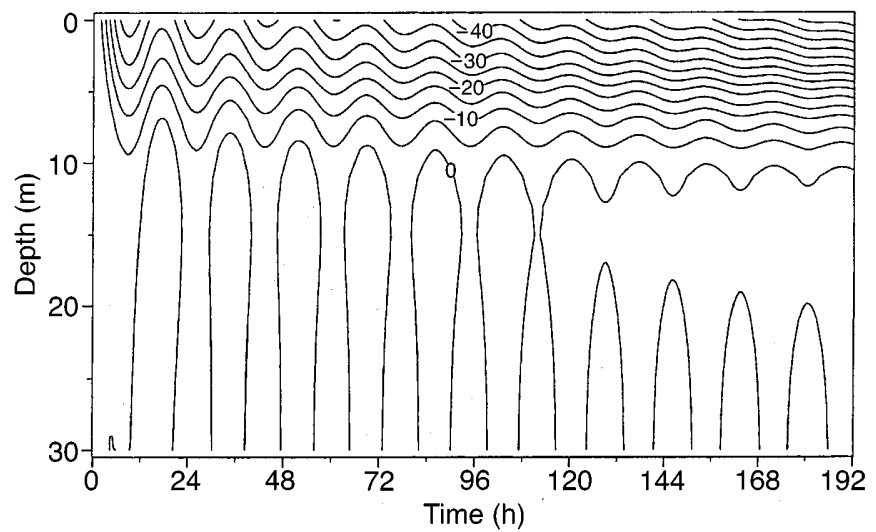

(c)

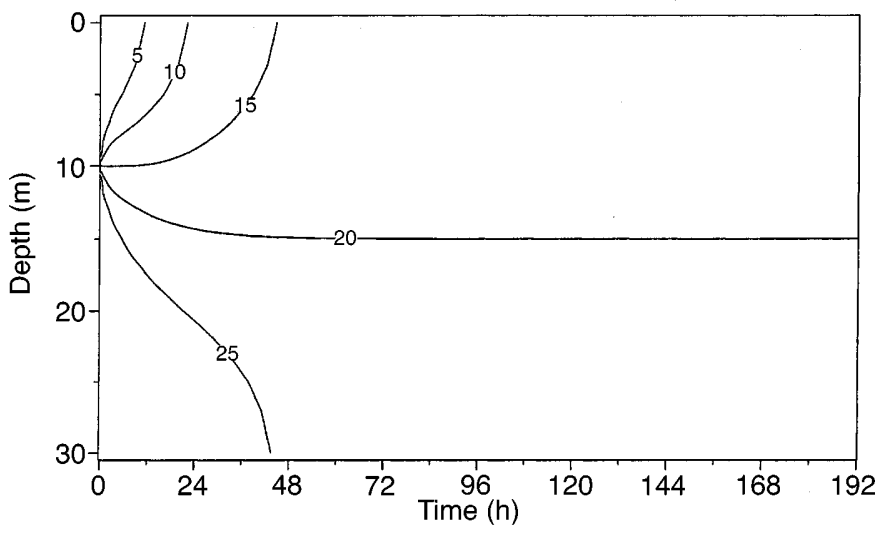

Fig. 2a-c. Solution of the initial simulation with a constant turbulent viscosity $\left(v_{t}=10 \mathrm{~cm}^{2} \cdot \mathrm{s}^{-1}\right)$. a Velocity $u\left(\mathrm{~cm} \cdot \mathrm{s}^{-1}\right), \mathbf{b}$ velocity $v\left(\mathrm{~cm} \cdot \mathrm{s}^{-1}\right)$, c salinity (psu). The contour intervals are 5 units for each variable plotted

terms which appear in the second members of Eqs. (25)(31) are then all equal to zero except for those corresponding to the chosen observations.

\subsection{Optimization results}

The most basic numerical experiment is to consider all the assimilated data $u, v, S$ as error free data. In comparison with a cost function formulation including a first guess (Yu and O'Brien, 1991), the decrease of the cost function $J$ is slower, but after about 3000 iterations, $J$ reaches almost zero. The optimized viscosity values (Fig. 3) provide fields of $u, v, S$ which appear to be indistinguishable from the observations. This results shows the feasibility of the method without using a first guess. Nevertheless, it must be recalled that such a good fit between model solution and data set is possible only because the data set was obtained via the complete $k+L$ model and so is fully consistent with the optimization constraints. This cannot be expected with in-situ experimental data.

Besides, the optimal set of turbulent viscosity values (Fig. 3) is sometimes different locally from the one given by the $k+L$ model used to construct the data set. It can be noticed that, even when different, the turbulent viscosity issued from the physical turbulence model and

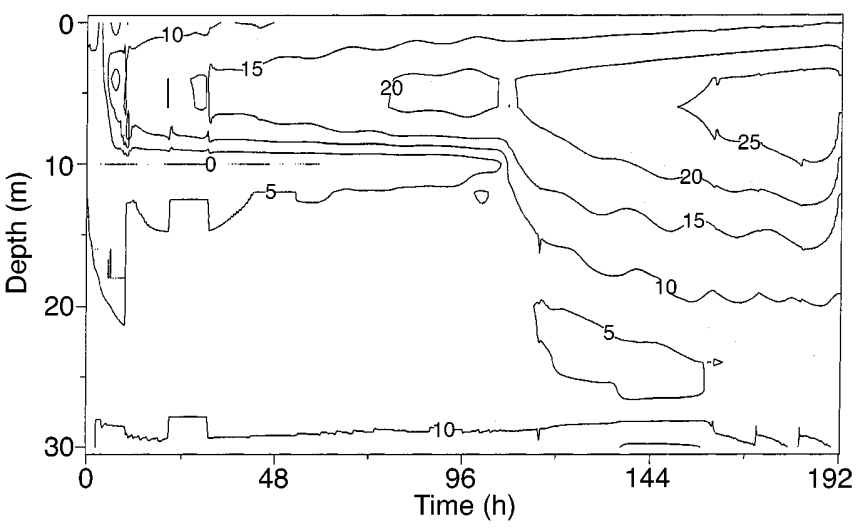

Fig. 3. Optimal turbulent viscosity $\left(\mathrm{cm}^{2} \cdot \mathrm{s}^{-1}\right)$. The contour intervals are $5 \mathrm{~cm}^{2} \cdot \mathrm{s}^{-1}$

the optimal viscosity issued from the data assimilation fitting can lead to the same numerical solution of the model. This paradoxical behaviour can be explained. The optimal viscosity has a degree of freedom (DoF) per grid point and time set while $v_{t}$, computed with the $k+L$ model, has a smaller number of DoF equal to the number of constants appearing in the $k+L$ turbulence model. In the optimization procedure, the turbulent viscosity value at a grid point and time step is not 
constrained by the global physical consistency due to the equation of the $k+L$ turbulence model. Furthermore, the sensitivity of a model to its parametrization may be so weak that the control of these parameters cannot be ensured. As already explained by Panchang and Richardson (1993), the eddy viscosity appear only in the local diffusion terms so that in weak vertical gradient regions, it can take any value without influencing the simulation results. This states the problem of the possibility to control models by parameters that do not have everywhere a real influence on the solution.

The ability of the method to recover the model control from a part of the observation data set must now be tested. In a second experiment, where only one variable, for instance $S$, is assimilated, the optimization procedure succeeded in recovering exactly the salinity field $(J=0)$ and the velocity fields (Fig. 4) with a better accuracy in stratified regions. The controllability problem leads to an optimal eddy viscosity that can take any possible value in zones of zero vertical salinity gradient. The uniqueness of the solution is then not ensured and the turbulent viscosity remains near its initial value (here $10 \mathrm{~cm}^{2} \mathrm{~s}^{-1}$ ). For practical purposes, the velocity field is then affected in these regions and it could be worse with another initial value like $0 \mathrm{~cm}^{2} \mathrm{~s}^{-1}$ or $100 \mathrm{~cm}^{2} \mathrm{~s}^{-1}$. The retrieval of the velocity field from the salinity field is interesting in itself since hydrological data are often more easily available than dynamic data. It must however be borne in mind that in the model the salt diffusivity is assumed to be proportional to the turbulent viscosity for the momentum diffusion ( $S c$ is a constant). Further generalization to models with eddy viscosity and diffusivity using other relationships should be made.

For the same reason, the assimilation of only one velocity component $u$ or $v$ leads to similar conclusions. $u$ and $v$ are in quadrature, and so give the same content of information for the optimization. The sudden event of the halocline break is included in the velocity field information. In fact, with a slower convergence rate compared with the previous experiment, the model solution is recovered even for the salinity.

The three previous numerical experiments were performed with a great number of DoF with good efficiency due to the ideal consistency of the data with the model. Unfortunately, oceanic observations are often sparse and noisy. In order to have a more realistic representation of in-situ observations, the following assimilation experiments are carried out with noisy and sparse data. To simulate different station measurements conducted from an oceanographic vessel, either hydrological or current profiling experi- (a)

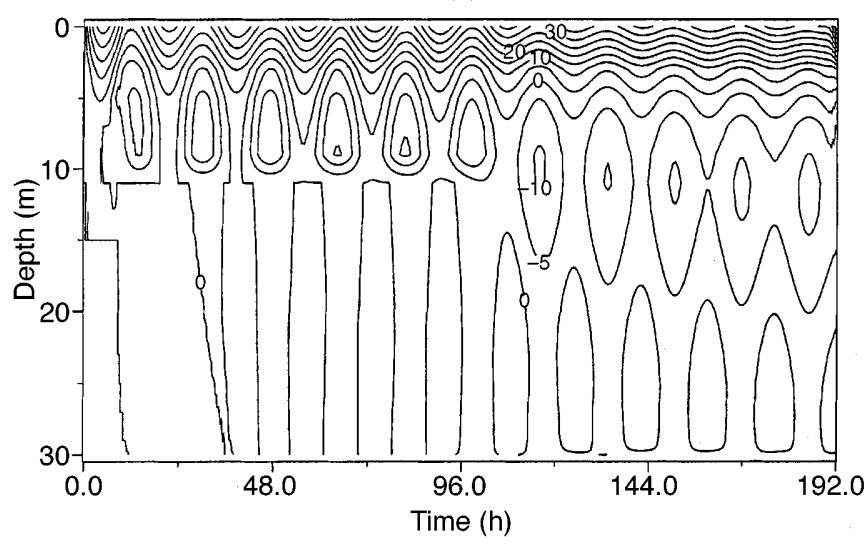

(c)

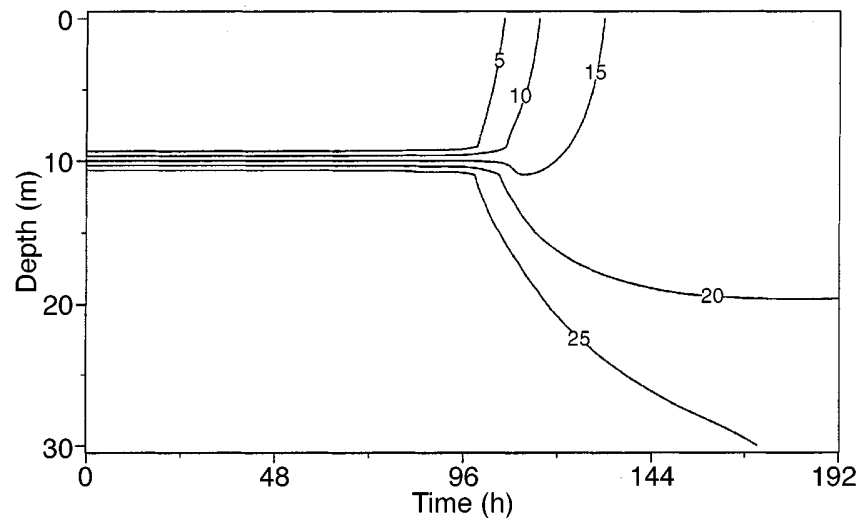

(b)

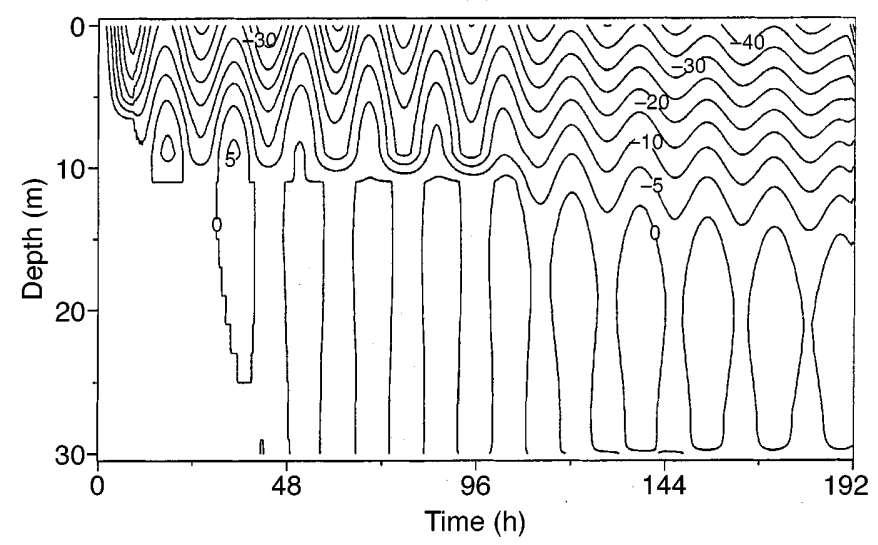

(d)

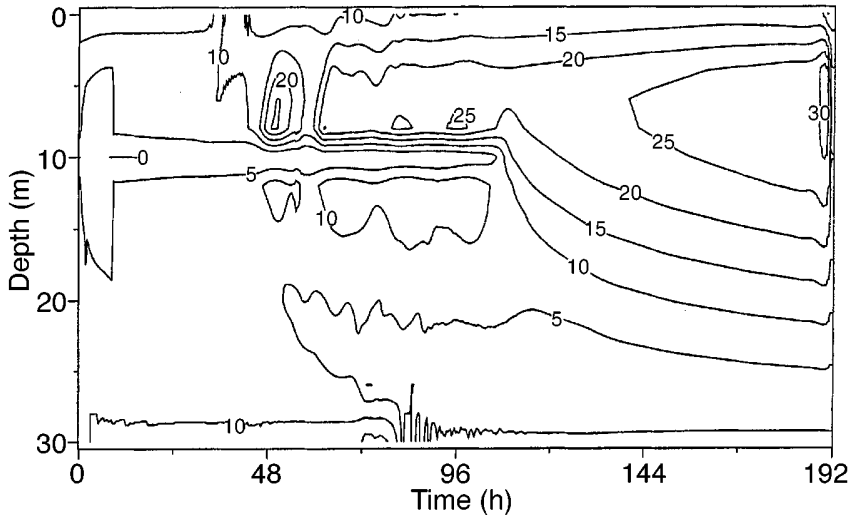

Fig. 4a-d. Optimal solution and optimal turbulent viscosity obtained by the assimilation of the salinity data. a Velocity $u\left(\mathrm{~cm} \cdot \mathrm{s}^{-1}\right), \mathbf{b}$ velocity $v\left(\mathrm{~cm}^{-1} \mathrm{~s}^{-1}\right)$, c salinity $(\mathrm{psu}), \mathbf{d}$ turbulent viscosity $\left(\mathrm{cm}^{2} \cdot \mathrm{s}^{-1}\right)$. The contour intervals are 5 units for each variable plotted 
ments, the proposed application consists in assimilating noisy velocity and salinity profiles taken at $48 \mathrm{~h}, 96 \mathrm{~h}$, $144 \mathrm{~h}$ and $192 \mathrm{~h}$. A white and gaussian noise is added with a rms equal to $6 \mathrm{~cm} \mathrm{~s}^{-1}$ for the velocities and 4 psu for the salinity. This rather severe noise has been chosen to test the robustness of the optimal control method. As the control dimension greatly oversizes the number of sparse data, the unique determination of an optimal time-dependent eddy viscosity cannot be expected. To keep the problem over-determined, the control dimension must be reduced at least to the same order as the number of data. Following the idea of Panchang and Richardson (1993), a penalty term (e.g. $\partial v_{t}(t) / \partial t$ ) could be added in the cost function to keep a continuous time-dependence of the viscosity. Here, a time-constant turbulent viscosity profile over each 48-h time-interval is chosen. The problem consists then in optimizing four viscosity profiles given for each period: 0 h-48 h, 48 h-96 h, 96 h-144 h, and 144 h-192 $\mathrm{h}$. The profile assimilation leads to the recovery of the full solution $u, v, S(z, t)$ (Fig. 5). One can note the time jumps of the solution corresponding to the optimal turbulent viscosity profiles. Figures 6 shows the $u, v, S$ optimal profiles at $t=96 \mathrm{~h}$ (the other time step results are not shown). Even if the assimilated profiles are noisy, the optimal profiles are very close to the true profiles issued from the full solution given by $k+L$ model. The initial starting profiles are computed with a constant viscosity value $\left(10 \mathrm{~cm}^{2} \mathrm{~s}^{-1}\right)$. Even with severe noise, the model is able to extract the mean information and to supply results provided with some physical consistency given by the model equations. The optimal eddy viscosity profiles (Fig. 7) evolve with time and with the wind increase. It is almost zero on the halocline for the two first profiles $(48 \mathrm{~h}, 96 \mathrm{~h})$ before it breaks. When the turbulent viscosity does not control the solution, its value remains near its initial starting value. The non-sequential nature of the proposed assimilation scheme must be underlined. Indeed, the information over the entire data recording is used as a whole to optimize the turbulent viscosity value at a grid point and time step. A sequential optimization would conversely consist in the separate processing of juxtaposed time periods with initial and final state observations. In the actual method, observations on past and future states are accounted for in addition to the initial and final states of the considered sequence. The case of a wrong data profile at a given time, say at $48 \mathrm{~h}$, which exhibits for instance a poor vertical resolution leading to an anticipated halocline break, could be corrected with later profiles where this discontinuity remains.

To summarise the previously described numerical experiment, it can be said that the assimilation technique turns out to offer a satisfactory robustness with noisy and sparse synthetic data. (a)

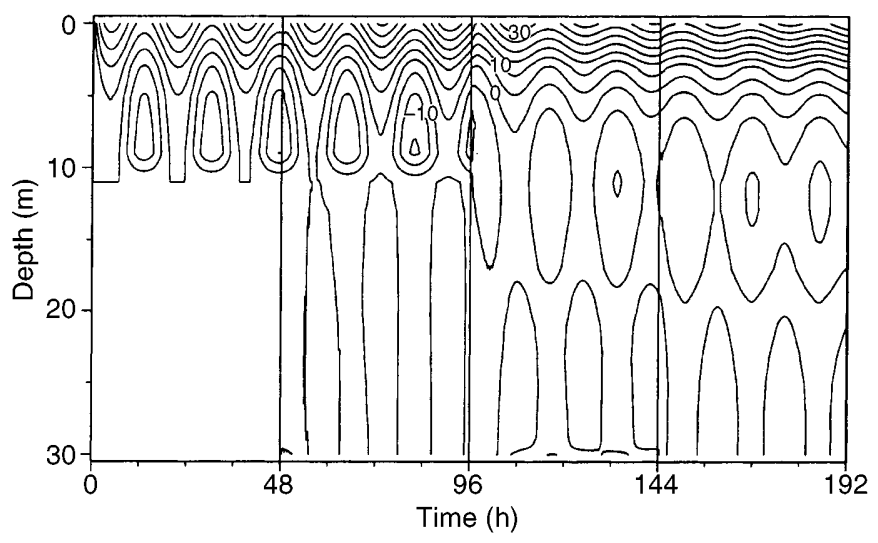

(b)

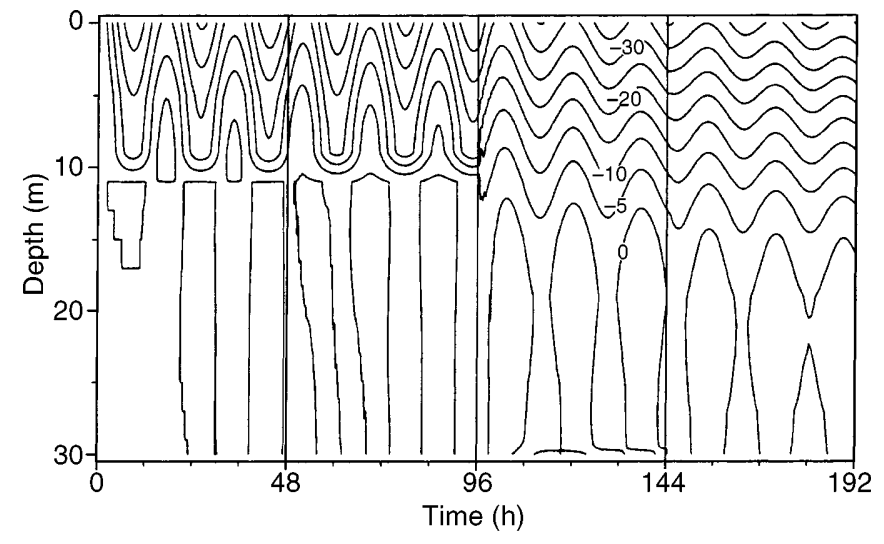

(c)

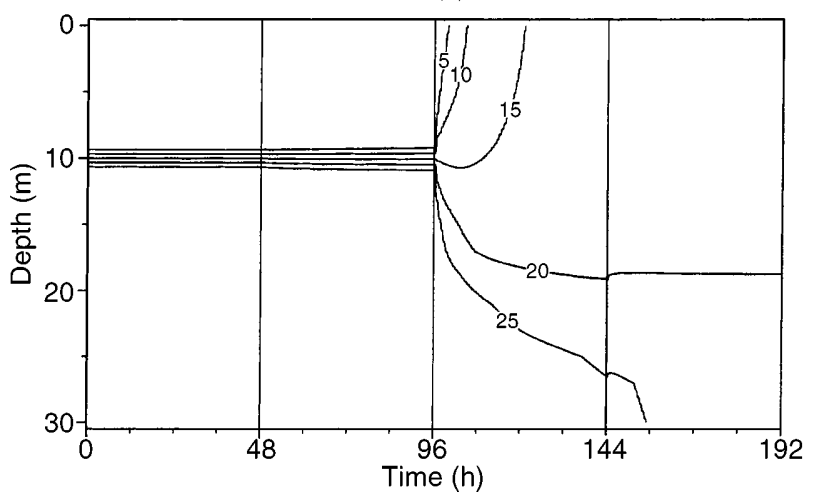

Fig. 5a-c. Optimal solution obtained by the assimilation of sequential and noised data ( $48 \mathrm{~h}, 96 \mathrm{~h}, 144 \mathrm{~h}$ and $192 \mathrm{~h})$. a Velocity $u\left(\mathrm{~cm} \cdot \mathrm{s}^{-1}\right)$, b velocity $v\left(\mathrm{~cm} \cdot \mathrm{s}^{-1}\right)$, c salinity (psu). The contour intervals are 5 units for each variable plotted 
(a)

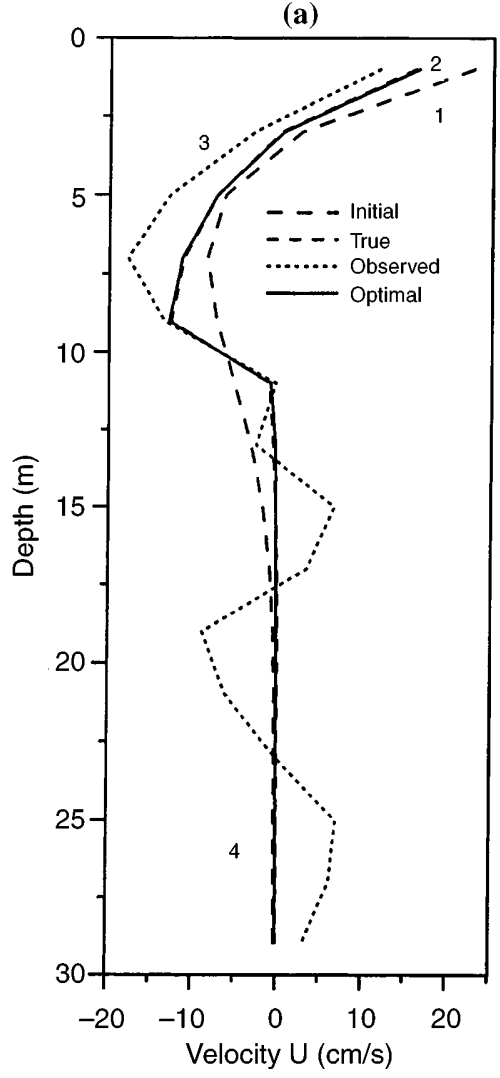

(b)

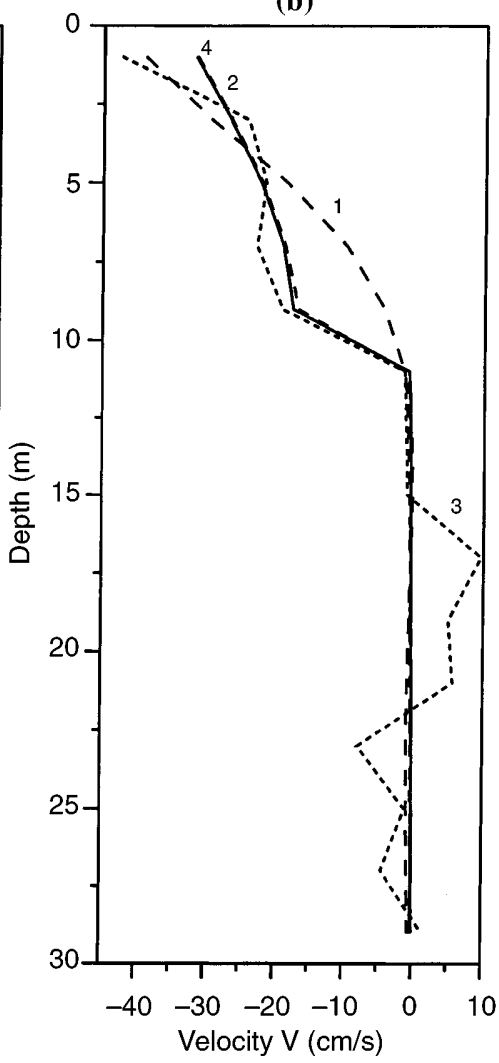

(c)

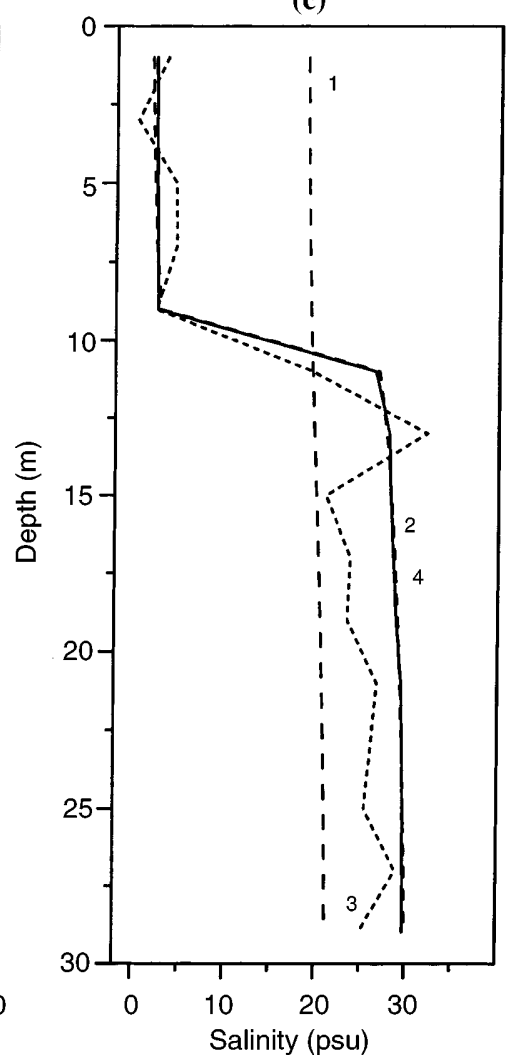

Fig. 6a-c. Velocity $u$ a, velocity $v \mathbf{b}$ and salinity $\mathbf{c}$ profiles at $t=48 \mathrm{~h}$. 1 , Profiles from the initial simulation computed with a constant turbulent viscosity $\left(v_{t}=10 \mathrm{~cm}^{2} \cdot \mathrm{s}^{-1}\right) .2$, True profiles. 3, Assimilated

profiles, the true profiles are noisy. 4, Optimal profiles, almost superimposed on the true ones

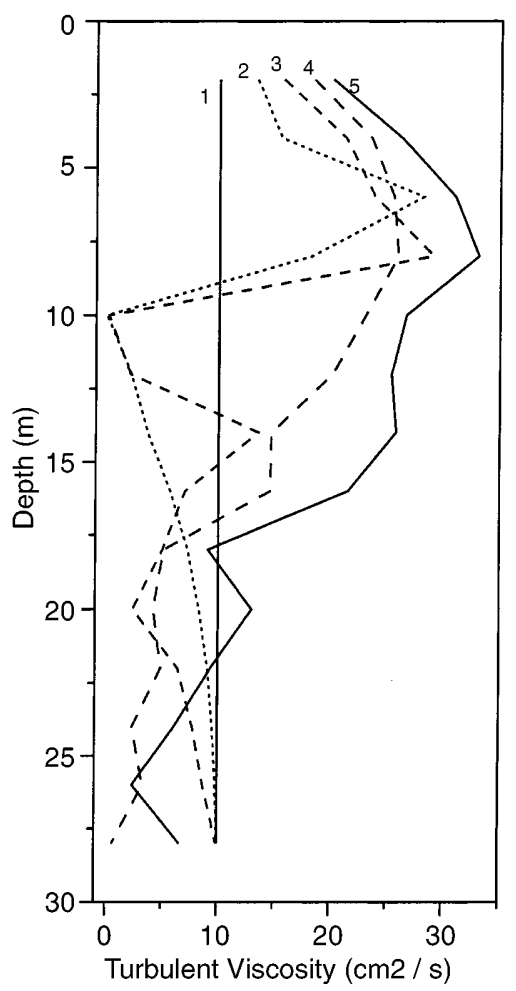

Fig. 7. Turbulent viscosity profiles. 1, Initial profile; Optimal profiles 2, 0 h-48 h; 3, 48 h-96 h; 4, 96 h-144 h; 5, 144 h-192 h

\section{A two space dimension optimization case}

The aim of this experiment is to show that the optimal control method can be used for more than one space dimension. The method described in Sect. 2.3 in the more general case has been applied in the previous section for only one space dimension and the time dimension. The control size was already very large in this previous simple case, involving a high numerical cost and a slow convergence of the method. This cost is enhanced by the need of the direct solution to solve the adjoint model: the values of the direct variables are to be stored in the direct path and read from the memory in the inverse path. This is why the control is here limited only to two space dimensions and considered as constant in time. In fact, a space dimension takes the place of the time dimension. For the same reason, a simplified theoretical model of ocean and of wind patterns are considered using a simple geometrical grid. The integration proceeds also for a relatively short simulation time. In addition it is the temperature distribution that here modifies the stratification.

The 2-D space-dependent viscosity optimization problem is exemplified on the simulation of a transient coastal upwelling assuming that $v_{t}$ is time-independent. The domain is closed to the west by a coast on which zero cross-shore transport conditions are specified. A 
constant wind $\left(5 \mathrm{~m} \mathrm{~s}^{-1}\right)$ is assumed to blow towards the north. By assuming the domain is open to the south and to the north, the solution does not vary along the direction $y$. By taking Neumann open ocean boundary conditions to the south and north, a two space dimension problem in a plane perpendicular to the coast is obtained. Neumann conditions are also imposed at the east boundary. The domain is extended to $40 \mathrm{~km}$ from the coast with a horizontal grid size $\Delta x=2 \mathrm{~km}$, whereas the depth is constant $(40 \mathrm{~m})$ with a vertical grid size $\Delta z=2 \mathrm{~m}$. The latitude is $45^{\circ} \mathrm{N}$, which leads to a Coriolis parameter $f=10^{-4} \mathrm{~s}^{-1}$. The time step is $\Delta t=50 \mathrm{~s}$ for a $48 \mathrm{~h}$ simulation. The model is started from a state of rest, a surface temperature of $25^{\circ} \mathrm{C}$ and a linear vertical gradient of temperature $\frac{\Delta T}{\Delta z}=0.5^{\circ} \mathrm{C} \mathrm{m}^{-1}$. Salinity is taken to be uniform and constant in time.

The observations are created from a 2-D steady viscosity field corresponding to the results given by the $k+L$ model at $t=48 \mathrm{~h}$. At this time, the turbulent viscosity field is nearly steady and so can be taken as the steady control to be recovered. Nevertheless, one can remark that, even if the viscosity is considered as constant in time, coastal upwelling phenomena is already not steady after $48 \mathrm{~h}$. During this transitional

(a)

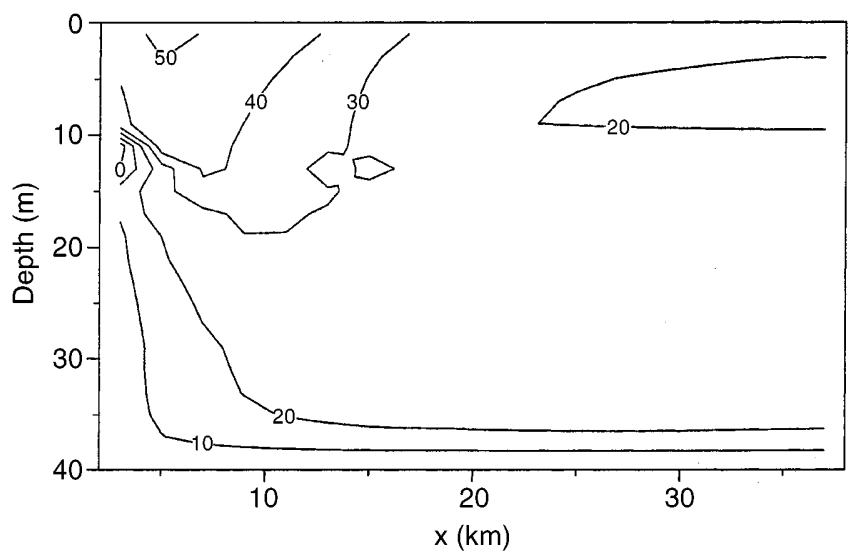

(c)

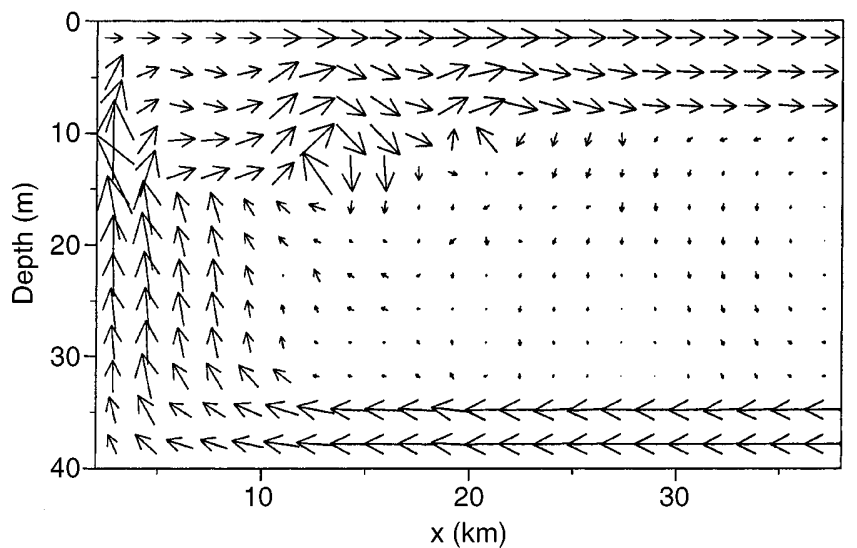

Fig. 8a-d. Solution of the direct simulation used to construct the observation of coastal upwelling at $t=48 \mathrm{~h} . x(\mathrm{~km})$ is the distance to the coast. a Velocity $v\left(\mathrm{~cm} \cdot \mathrm{s}^{-1}\right)$, b Temperature $\left({ }^{\circ} \mathrm{C}\right)$, c vertical period, inertial waves always propagate (see Crepon and Richez, 1982, or Kundu et al., 1983, for more details). The model therefore gives results as expected (e.g. Foo, 1981). Close to the coast, nearly $5 \mathrm{~km}$, a coastal jet expands in the direction of the wind. The surface mixing layer is advected towards the open sea, whereas the return current stays between the boundaries of the bottom mixing layer. Near the coast, cold waters are advected from the bottom to the surface. Figure 8 presents the solution $\left(u, v, w, T, v_{t}\right)$ at the end of the observations simulation.

The aim is then to reconstruct the 2D space-dependent turbulent viscosity field from the solution of the model. The initial conditions (state of rest and linear temperature stratification) are presumed to be known. The initial solution obtained with an uniform viscosity value of $10 \mathrm{~cm}^{2} \mathrm{~s}^{-1}$ (Fig. 9) is considered as the starting point of the optimization procedure. The results obtained for this initial simulation show that it is necessary to consider a spatial variability of the viscosity.

In order to elaborate the problem and make the method more adapted to usual practical oceanographic application, only temperature data are assimilated, assuming the velocity field to be unknown. As the

(b)

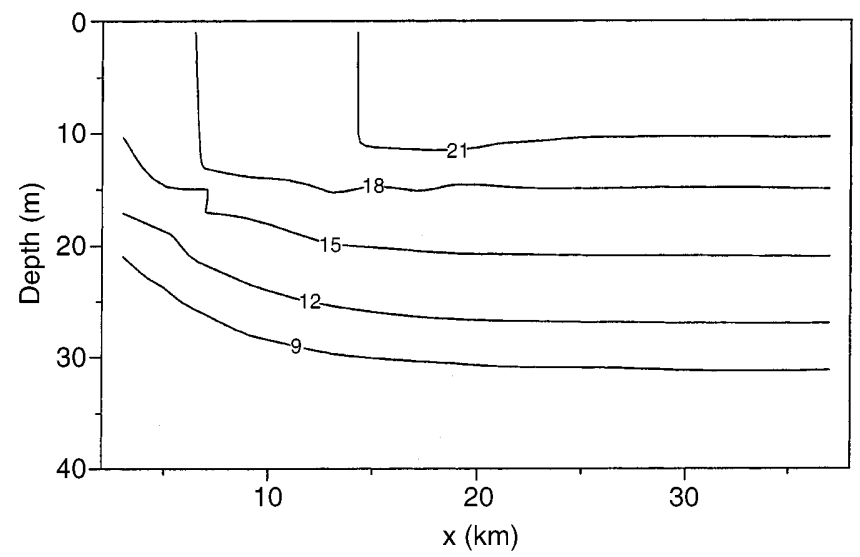

(d)

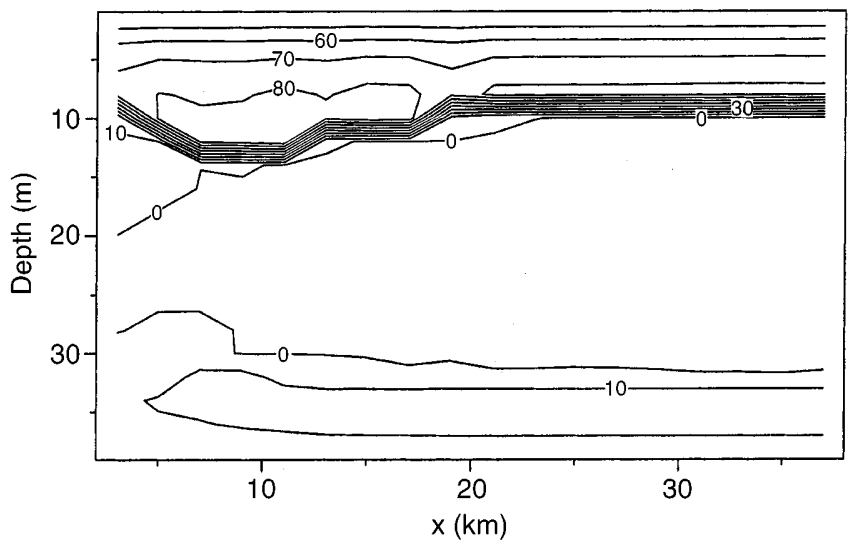

current vector $\vec{U}=\left(u, w \times 10^{3}\right)$, the maximum length is $19 \mathrm{~cm} \mathrm{~s}^{-1}, \mathbf{d}$ turbulent viscosity $\left(\mathrm{cm}^{2} \cdot \mathrm{s}^{-1}\right)$ 
(a)

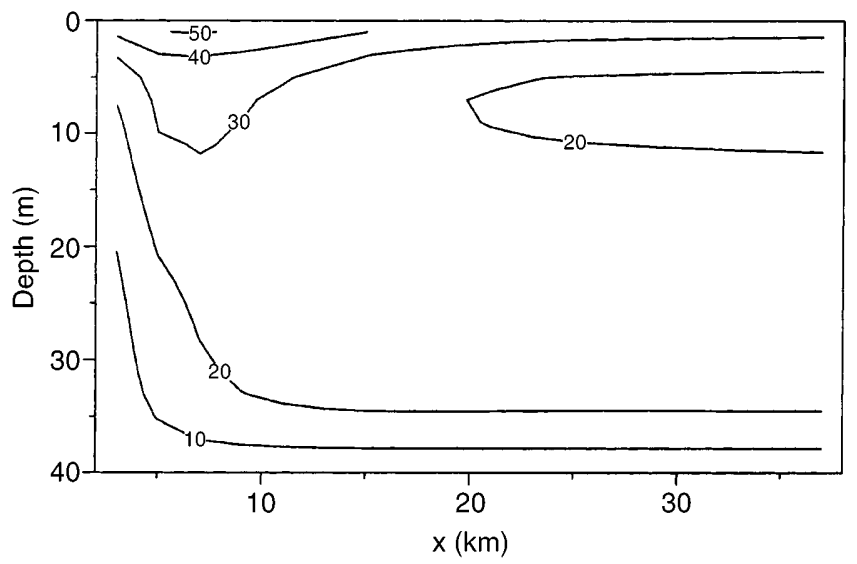

(b)

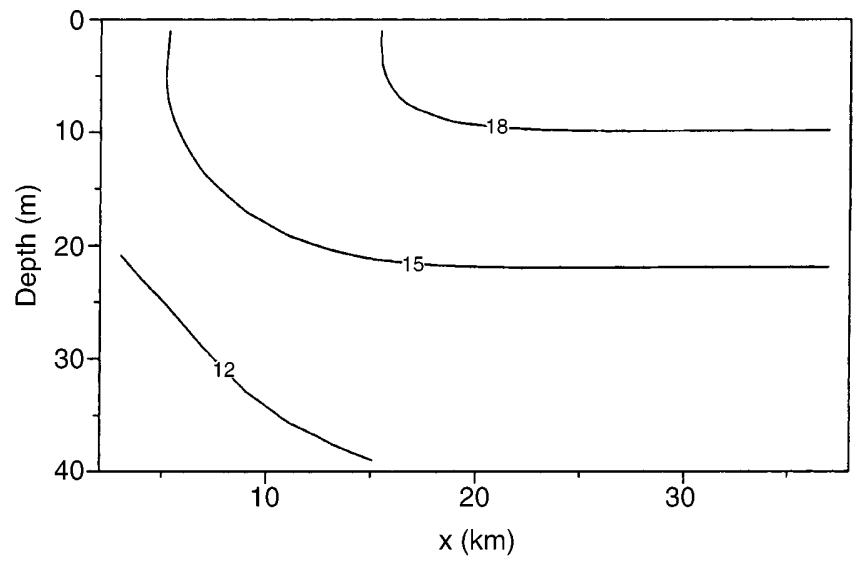

(c)

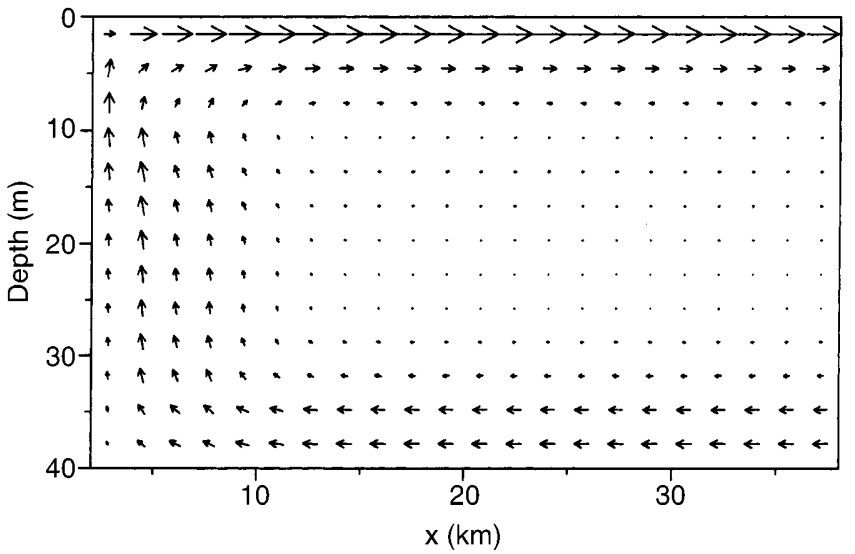

Fig. 9a-c. Solution of the initial simulation of a coastal upwelling with a constant turbulent viscosity $\left(v_{t}=10 \mathrm{~cm}^{2} \cdot \mathrm{s}^{-1}\right)$ at $t=48 \mathrm{~h} . x(\mathrm{~km})$ is the distance to the coast. a velocity $v\left(\mathrm{~cm} \cdot \mathrm{s}^{-1}\right), \mathbf{b}$ temperature $\left({ }^{\circ} \mathrm{C}\right)$, c vertical current vector $\vec{U}=\left(u, w \times 10^{3}\right)$, the maximum length is $17 \mathrm{~cm} \mathrm{~s}^{-1}$

control is considered as constant in time, a single time sequence of data is needed.

First, it has been chosen to assimilate the complete and noise-free temperature field at $t=48 \mathrm{~h}$. The number of data is almost equal to the number of parameters and it is already a significant difficulty to suppose that no information is known for the velocity field and that no other data are available between $0 \mathrm{~h}$ and $48 \mathrm{~h}$.

The optimal control method provides by inversion a viscosity field which leads to a quite accurate simulation of the temperature field. One can remark that, as well as with the salinity data in the previous example, the only assimilation of the temperature data allows to reconstruct the dynamics. Figure 10 presents this complete solution, very close to the simulated observations.

Secondly, in order to test the robustness of the method, the previous experiment is realised assimilating the complete but noise-distributed temperature field at $t=48 \mathrm{~h}$. A white and gaussian noise is added to the temperature field. The level of noise is raised step by step as long as the method remains efficient enough. The deduced borderline case, presented here, corresponds to a noise with a rms equal to $2{ }^{\circ} \mathrm{C}$, which is more higher than the in-situ measurement errors occurring with usual devices, such as CTD profilers. Figure 11 shows the assimilated temperature field.

It can be seen that the added noise is smoothed by the physics of the model which acts as a strong constraint. The discrepancies of the data with the model, induced by a gaussian and white noise, remains weak enough to allow the method to work as shown in Fig. 12. This 2-D optimization case will allow us to consider more complex situations in the future.

\section{Summary and conclusions}

As an alternative to classical turbulence modelling, the data assimilation method of optimal control is formulated to optimize the turbulent viscosity in a 3-D primitive equation model of the ocean circulation. The chosen model and its full turbulence formulation $(k+L)$ is described and used to simulate synthetic observations (velocity, salinity or temperature) of two wind-driven events: the first concerns an open stratified ocean, and the second, a coastal upwelling. These data are assimilated to reconstruct the turbulent viscosity field, which depends either on time and 1-D space or 2-D space. An 
(a)

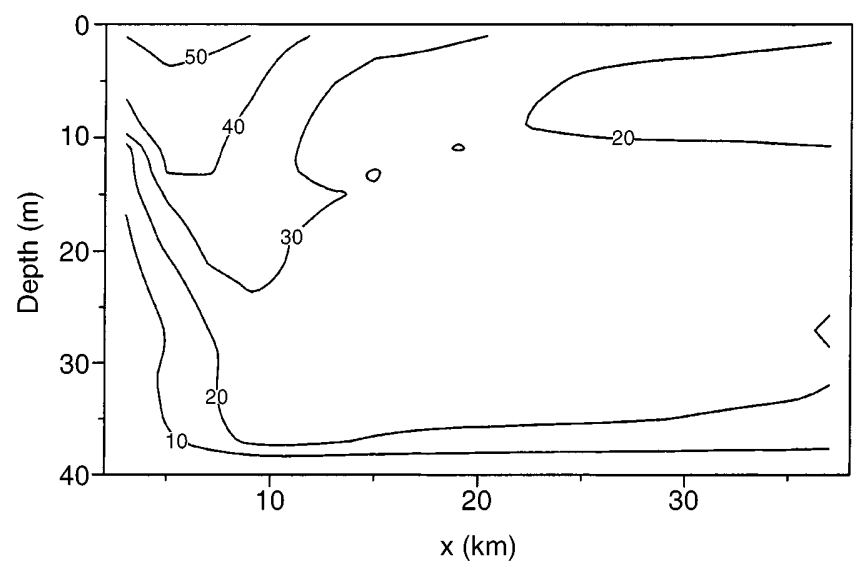

(c)

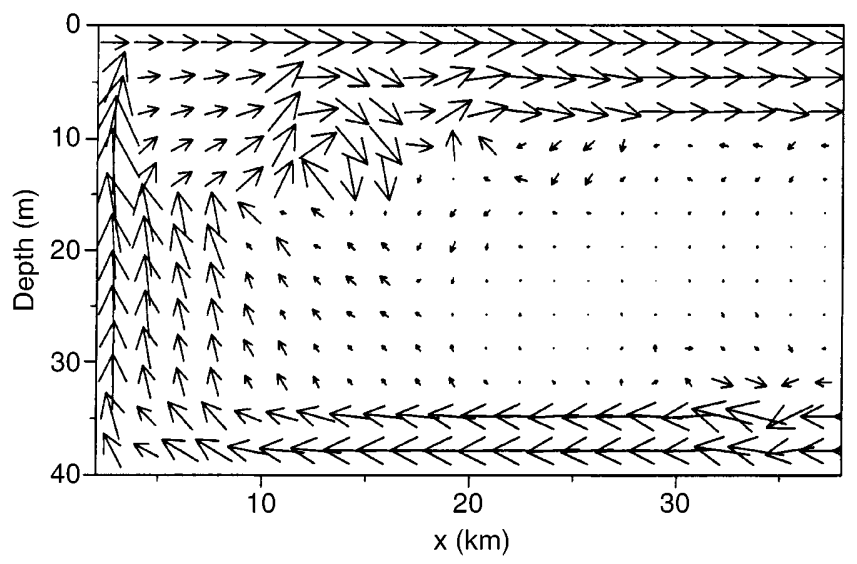

Fig. 10a-d. Optimal solution and optimal turbulent viscosity obtained by the assimilation of the temperature data of the coastal upwelling. $x(\mathrm{~km})$ is the distance to the coast. a Velocity $v\left(\mathrm{~cm} \cdot \mathrm{s}^{-1}\right), \mathbf{b}$

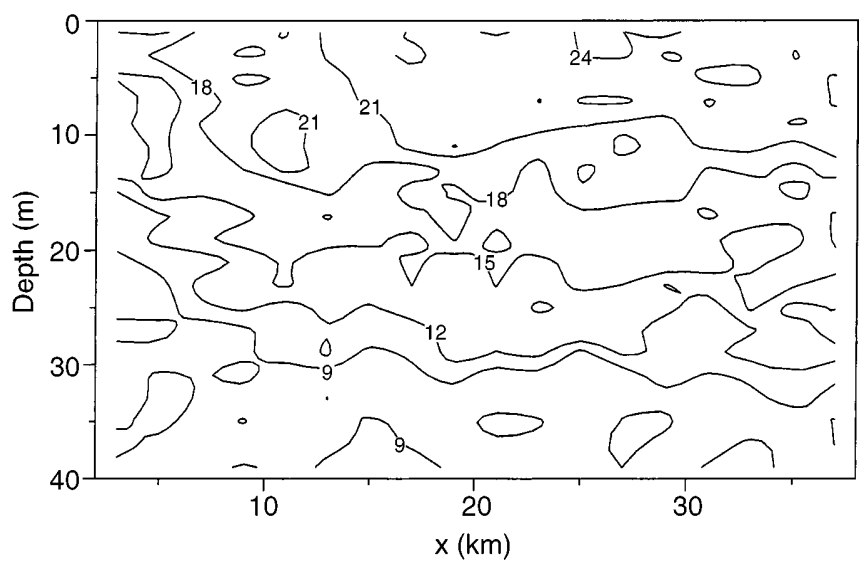

Fig. 11. Assimilated and noisy temperature data $\left({ }^{\circ} \mathrm{C}\right)$ of the coastal upwelling, at $t=48 \mathrm{~h}$, obtained by addition of a white and gaussian noise with an rms equal to $2{ }^{\circ} \mathrm{C}$

adjoint model of the direct model (without the complete turbulence formulation) is integrated backward in time to compute the gradient of the cost function with respect to the control and therefore performs the minimization. (b)

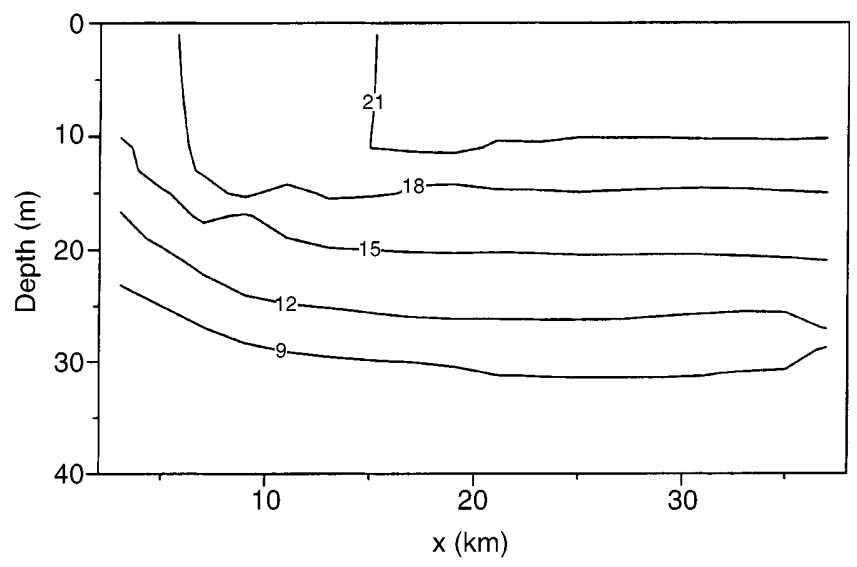

(d)

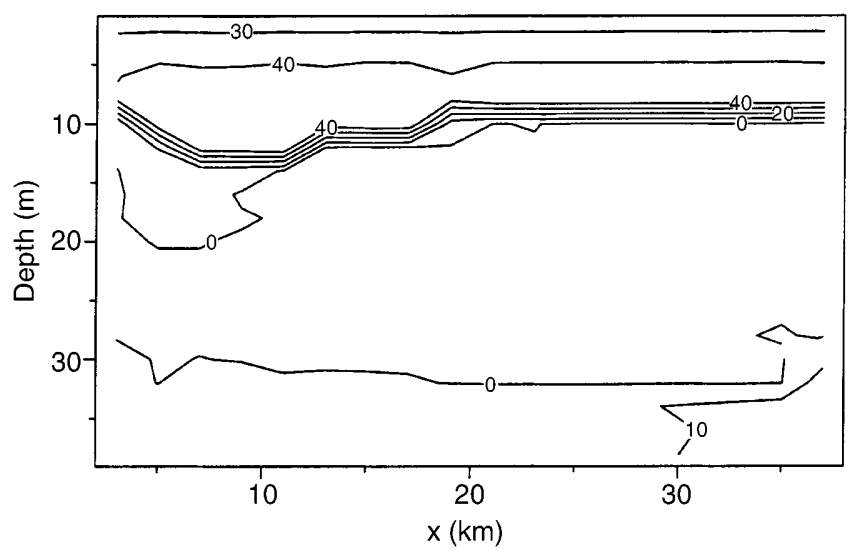

Temperature $\left({ }^{\circ} \mathrm{C}\right)$, c vertical current vector $\vec{U}=\left(u, w \times 10^{3}\right)$, the maximum length is $14 \mathrm{~cm} \mathrm{~s}^{-1}$, $\mathbf{d}$ turbulent viscosity $\left(\mathrm{cm}^{2} \cdot \mathrm{s}^{-1}\right)$

The interest of fitting the vertical turbulent mixing via $v_{t}$ must be stressed. In many coupled physicobiogeochemical models (e.g. Pinazo et al., 1996), the turbulent state input is only described by a distribution of the eddy vertical viscosity. Its optimization by data assimilation could be useful in this case, the detailed understanding of the turbulent processes not being required. Of course, the success of the method is conditioned by the nature of the data. In the current work, it is pointed out that a vertical mixing process can be recovered using only salinity or temperature data. Furthermore, it would also be interesting to apply this method to reconstruct a physical mixing event from the observations of a conservative tracer (Vandenberghe, 1992).

The optimal control method has shown its efficiency in reconstructing the turbulent viscosity field from synthetic velocity or salinity data. Next, as has already been done on a time-independent 1-D optimization in an Ekman model (Yu and O'Brien, 1991), the optimization in a 3-D primitive equation model will have to be tested with assimilation of real observations. This preliminary study has pointed out the difficulties that could be encountered. 
(a)

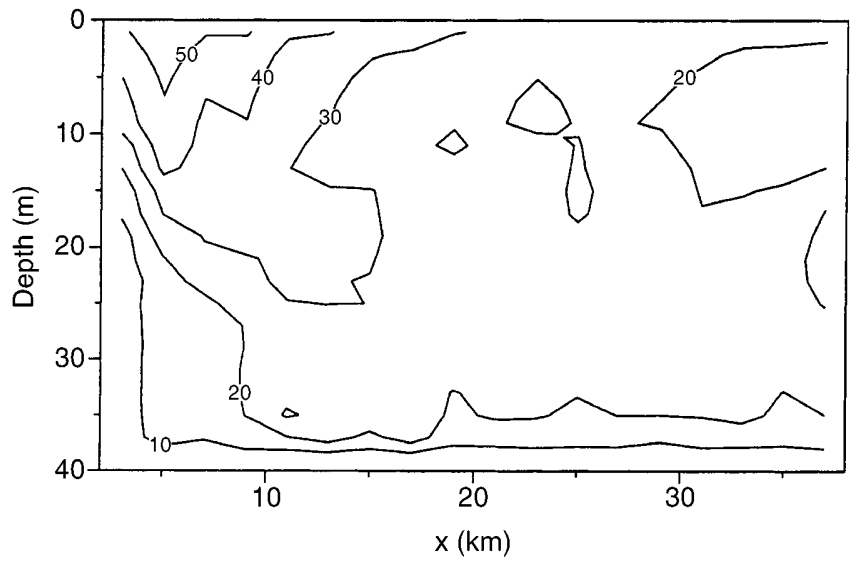

(c)

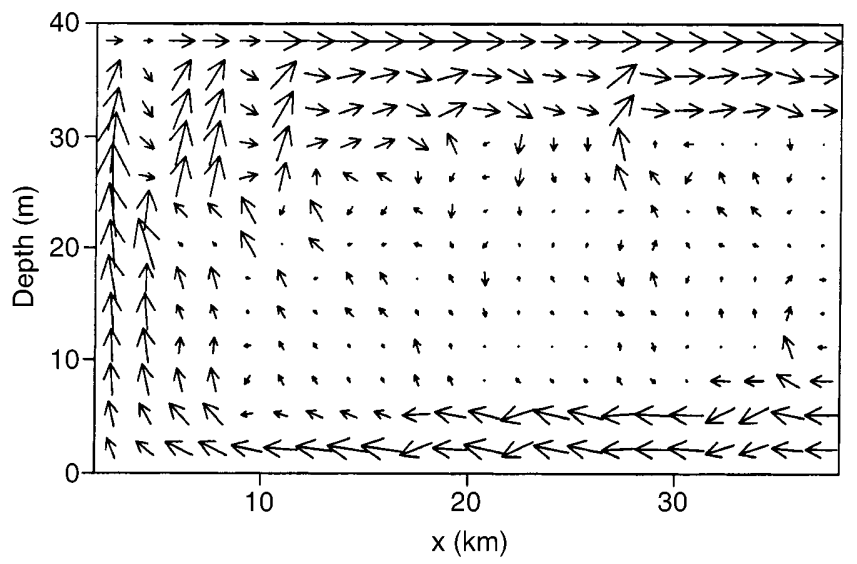

Fig. 12a-d. Optimal solution and optimal turbulent viscosity obtained by the assimilation of the noisy temperature data of the coastal upwelling. $x(\mathrm{~km})$ is the distance to the coast. a Velocity $v\left(\mathrm{~cm} \cdot \mathrm{s}^{-1}\right), \mathbf{b}$

The first problem is the large dimension of the control vector. This involves an important numerical cost and a slow convergence rate of the method. Then, a number of data oversizing the control dimension is required. The eddy viscosity variations should be chosen versus the available data. For example, in Sect. 4, as only initial and final states are known, the eddy viscosity is optimized as a time-constant. The full time-space 4-D variational formalism has been described and should be adapted for each physical situation and assimilable data.

The second problem is the controllability problem. Prior knowledge of the distributed turbulent viscosity is sometimes not available, especially for a time and space dependent problem, and the method must be investigated without first guess. As the eddy viscosity is not always an influential parameter in the calculations, its uniqueness may not be ensured in some areas. Uniqueness in these zones could be achieved using a first guess but with a loss of accuracy of the model results in the strong eddy viscosity influenced regions. Moreover, the optimal viscosity can then take any values which appear sometimes to be physically inconsistent. In the optimization procedure the zero order turbulence closure model allows a degree of freedom by grid point and (b)

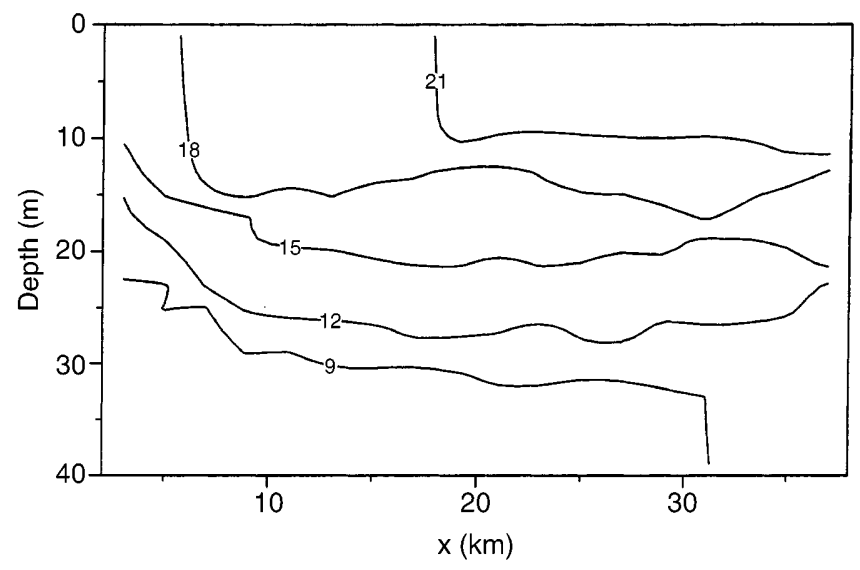

(d)

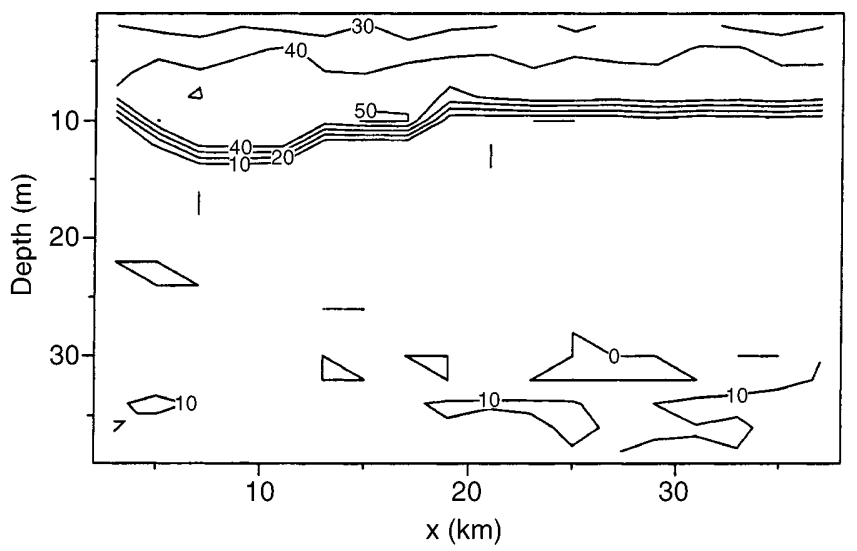

temperature $\left({ }^{\circ} \mathrm{C}\right)$, c vertical current vector $\vec{U}=\left(u, w \times 10^{3}\right)$, the maximum length is $14 \mathrm{~cm} \mathrm{~s}^{-1}$, $\mathbf{d}$ turbulent viscosity $\left(\mathrm{cm}^{2} \cdot \mathrm{s}^{-1}\right)$

time step. In contrast, in the $k+L$ turbulence model, the viscosity is strongly constrained by the $k$ and $L$ via Eq. (10), and so has a smaller number of degrees of freedom equal to the number of constants (e.g. $\left.C_{v}, k, \alpha, \varepsilon_{0}\right)$. In this case, turbulence model calibration is required. It could be viewed as a challenging problem to consider in turn these parameters as controls of the model to be optimized. However, hardly any difficulties are probably to be expected due to the strong non linearities of the dependence between the model solution and these constants.

Acknowledgements. Topical Editor D. Y. Webb thanks A. Ochlies and another referee for their help in evaluating this paper.

\section{References}

Andre, J. C., G. De Moor, P. Lacarrere, G. Therry and R. Du Vachat, Clipping approximation and inhomogeneous turbulence simulations in, Turbulent shear flows I, Springer, Berlin Heidelberg New York, 307-318, 1979.

Begis, D., and M. Crepon, On the generation of currents by winds: an identification method to determine oceanic parameters, Lecture notes in Physics, 58, 1975. 
Blackadar, A. K., The vertical distribution of wind and turbulent exchange in a neutral atmosphere, J. Geophys. Res., 4,333-355, 1962.

Blumberg, A. F., and D. M. Goodrich Modelling of wind-induced destratification in Chesapeake Bay, Estuaries, 13(3) 236-249, 1990.

Bennett, A. F., Inverse methods in physical oceanography, Cambridge University Press, Cambridge, UK, 346 pp, 1992.

Bennett, A. F. and R. N. Miller, Weighting initial conditions in variational assimilation schemes, Mon. Weather Rev., 119, 1098-1102, 1991.

Crépon, M. and C. Richez, Transient upwelling generated by twodimensional atmospheric forcing and variability in the coastline, J. Phys. Oceanogr., 12, 1437-1456, 1982.

Courtier, P., and O. Talagrand, Variational assimilation of meteorological observations with the direct and adjoint shallow-water equations, Tellus, 42A, 531-549, 1990.

Devenon, J. L., Optimal control theory applied to an objective analysis of a tidal current mapping by HF radar, J. Atmos. Ocean. Tech., 7, 269-284, 1990.

Eknes, M., and G. Evensen, Parameter estimation solving a weak constraint variational formulation for an Ekman model, J. Geophys. Res., 102 (C6), 12: 479-491, 1997.

Foo E. C., A two-dimensional diabatic isopycnal model simulating the coastal upwelling front, J. Phys. Oceanogr., 11, 604-626, 1981.

Gilbert, J. C., and C. Lemarechal, Some numerical experiments with variable-storage quasi-Newton algorithms, Math. Progr., 45, 407-435, 1989.

Kundu, P. K., S. Y. Chao, and J. P. McCreary, Transient coastal currents and inertio-gravity waves, Deep-Sea Res., 30, 10A: 1059-1082, 1983.

Le Dimet, F.-X, and O. Talagrand, Variational algorithms for analysis and assimilation of meteorological observations: theoretical aspects, Tellus, 38A, 97-110, 1986.

Leendertse, J. J., and S.-K. Liu, A three-dimensional model for estuaries and coastal seas: turbulent energy computation, The RAND Corporation (California), Internal Rep., 1977.

Leendertse, J. J., R. C. Alexander and S.-K. Liu, A threedimensional model for estuaries and coastal seas: principles of computation, The RAND Corporation (California), Internal Rep., 1973.

Lellouche, J. M., Méthodes numériques d'assimilation de données appliquées à la modélisation tridimensionnelle des écoulements en milieu peu profond, PhD Thesis, Université d'Aix-Marseille II, $162 \mathrm{pp}, 1995$.

Lellouche, J. M., J. L., Devenon, and I., Dekeyser, Boundary control on Burgers' equation A numerical approach, Comput. Math. Applic., 28, 5: 33-44, 1994.

Lellouche, J. M., J. L. Devenon and I. Dekeyser, Data assimilation by optimal control in a 3-D coastal oceanic model: the problem of the discretization, J. Atmos. Ocean. Tech., 15, 2: 470-481, 1998.

Leredde, Y., Méthode d'assimilation de données par contrôle optimal appliquée à l'estimation des paramètres de diffusion turbulente dans un modèle $3 \mathrm{D}$ de circulation océanique, $\mathrm{PhD}$ Thesis, Université d'Aix-Marseille II, 149 pp, 1999.

Leredde, Y., J. M. Lellouche, J. L. Devenon and I. Dekeyser, On initial, boundary conditions and viscosity coefficient control for
Burgers' equation., Int. J. Num. Meth. Fluids, 28, 113-128, 1998.

Lions, J. L., Optimal control of system governed by partial differential equations, Springer, Berlin Heidelberg New York, 396 pp, 1971

Luyten, P. J., E. Deleersnijder, J. Ozer and K. G. Ruddick, Presentation of a family of turbulence closure models for stratified shallow water flows and preliminary application to the Rhine Outflow region, Cont. Shelf Res., 16, 1: 101-130, 1996.

Mellor, G. L., and T. Yamada, A hierarchy of turbulence closure models for planetary boundary layers, J. Atmos. Sci., 31, 17911806, 1974.

Mellor, G. L., and T. Yamada, Development of a turbulence closure model for geophysical fluid problems, Rev. Geophys. Space. Phys., 20, 851-875, 1982.

Menke, W., Geophysical data analysis: discrete inverse theory. Academic Press, London, 260 pp, 1984.

Moore, A. M., Data assimilation in a quasi-geostrophic openocean model of the Gulf Stream region using the adjoint method, J. Phys. Oceanogr., 21, 398-427, 1991.

Munk, W. H., and E. R. Anderson, Notes on a theory for a thermocline, J. Mar. Res., 7, 276-295, 1948.

Nihoul, J. C. J., Modèles Mathématiques et dynamique de l'environnement, ELE, Liège, 198pp, 1977.

Nihoul, J. C. J., E. Deleersnijder and S. Djenidi, Modelling the general circulation of shelf seas by $3 \mathrm{D} k-\varepsilon$ models, Earth-Sci. Rev., 26, 163-189, 1989.

Panchang, V. G., and J. E. Richardson, Inverse adjoint estimation of eddy viscosity for coastal flow models, J. Hydraulic Eng., 119, 4: 506-524, 1993.

Pedlosky, J., Geophysical fluid dynamics, Springer, Berlin Heidelberg New York, 624 pp., 1982.

Pinazo, C., P. Marsaleix, B. Millet, C. Estournel and R. Véhil, Spatial and temporal variability of phytoplankton biomass in upwelling areas of the northwestern Mediterranean: a coupled physical and biogeochimical modelling approach, J. Mar. Syst., 7,161-191, 1996.

Rodi, W., Turbulence models and their applications in hydraulics. A state of are review. International Association for Hydraulics Research, Delft, Netherlands, 104 pp, 1980.

Roquet, H., S. Planton and P. Gaspar, Determination of ocean surface heat fluxes by a variational method, J. Geophys. Res., 98(C6), 10: 211-221, 1993.

Seiler, U., Estimation of open boundary conditions with the adjoint method, J. Geophys. Res., 98, C12: 22, 855-22, 870, 1993.

Thouvenin, B., and J. C. Salomon, Modèle tridimensionnel de circulation et de dispersion en zone côtière à marée. Premiers essais: cas schématiques et baie de Seine, Oceanol. Acta, 7(4), 417-429, 1984.

Van Dam, G. C., and R. A. Louwersheimer, A three-dimensional transport model for dissolved and suspended matter in estuaries and coastal seas, in Dynamics and exchanges in estuaries and the coastal zone, Coastal and Estuarine Studies 40 A.G.U. Washington, DC, pp. 481-506 Ed., D. Prandle 1992.

Vandenberghe, F., Assimilation de measures satellitaires dans les modèles numériques par méthodes de contrôle optimal, $\mathrm{PhD}$ Thesis, ENSM Paris, 1992.

Yu, L., and J. J. O'Brien, Variational estimation of the wind stress drag coefficient and the oceanic eddy viscosity profile, J. Phys. Oceanogr. 21, 709-719, 1991. 